\title{
A novel quasi-exactly solvable spin chain with nearest-neighbors interactions
}

\author{
A. Enciso, F. Finkel, A. González-López*, M.A. Rodríguez \\ Departamento de Física Teórica II, Universidad Complutense, 28040 Madrid, Spain
}

Received 23 April 2007; accepted 2 July 2007

Available online 30 July 2007

\begin{abstract}
In this paper we study a novel spin chain with nearest-neighbors interactions depending on the sites coordinates, which in some sense is intermediate between the Heisenberg chain and the spin chains of Haldane-Shastry type. We show that when the number of spins is sufficiently large both the density of sites and the strength of the interaction between consecutive spins follow the Gaussian law. We develop an extension of the standard freezing trick argument that enables us to exactly compute a certain number of eigenvalues and their corresponding eigenfunctions. The eigenvalues thus computed are all integers, and in fact our numerical studies evidence that these are the only integer eigenvalues of the chain under consideration. This fact suggests that this chain can be regarded as a finite-dimensional analog of the class of quasi-exactly solvable Schrödinger operators, which has been extensively studied in the last two decades. We have applied the method of moments to study some statistical properties of the chain's spectrum, showing in particular that the density of eigenvalues follows a Wigner-like law. Finally, we emphasize that, unlike the original freezing trick, the extension thereof developed in this paper can be applied to spin chains whose associated dynamical spin model is only quasi-exactly solvable.
\end{abstract}

(C) 2007 Elsevier B.V. All rights reserved.

PACS: 75.10.Pq; 03.65.Fd

Keywords: Spin chains; Quasi-exact solvability; Calogero-Sutherland models; Freezing trick

\footnotetext{
* Corresponding author.

E-mail address: artemio@ fis.ucm.es (A. González-López).
} 


\section{Introduction}

Solvable spin chains have enjoyed a growing popularity in the last few years, due in part to their novel applications to SUSY Yang-Mills and string theories [1-6]. The prime example of such chains is the celebrated Heisenberg model describing $N$ spins in a one-dimensional lattice with isotropic nearest-neighbors interactions independent of the site. The Hamiltonian of the model is given by [7]

$$
\mathcal{H}_{\mathrm{He}}=\sum_{i} \mathbf{S}_{i} \cdot \mathbf{S}_{i+1}
$$

where $\mathbf{S}_{i}=\left(S_{i}^{x}, S_{i}^{y}, S_{i}^{z}\right)$ is the spin operator of the $i$ th site, the sum runs from 1 to $N$ (as always hereafter), and $\mathbf{S}_{N+1}=\mathbf{S}_{1}$. As is well known, for spin 1/2 the model (1) can be exactly solved using Bethe's ansatz [8-10]. Several (partially) solvable generalizations of the Heisenberg chain (1) with short range interactions (at most between next to nearest neighbors) have been subsequently proposed in the literature. These include, in particular, the family of chains with arbitrary spin and nearest-neighbors interactions polynomial in $\mathbf{S}_{i} \cdot \mathbf{S}_{i+1}$ of Refs. [11,12], as well as several models whose ground state can be written in terms of "valence bonds" $[13,14]$.

A different type of solvable spin chain with long-range position-dependent couplings was introduced independently by Haldane [15] and Shastry [16]. This chain describes a system of $N$ spins equally spaced on a circle, such that the strength of the interaction between each pair of spins is inversely proportional to their chord distance. The motivation for introducing the HS chain (2) was the fact that its exact ground state coincides with Gutzwiller's variational wave function for the Hubbard model [17-19] when the strength of the on-site interaction tends to infinity. We shall write the Haldane-Shastry (HS) Hamiltonian as

$$
\mathcal{H}_{\mathrm{HS}}=\frac{1}{2} \sum_{i<j} \sin \left(\vartheta_{i}-\vartheta_{j}\right)^{-2}\left(1-S_{i j}\right), \quad \vartheta_{i} \equiv \frac{i \pi}{N},
$$

where $S_{i j}$ is the operator exchanging the $i$ th and $j$ th spins. Although the particles' spin in the original HS chain was assumed to be $1 / 2$, one can more generally consider particles with $n$ internal degrees of freedom transforming under the fundamental representation of $\operatorname{su}(n)$. In this case the spin permutation operators can be written in terms of the fundamental $\operatorname{su}(n)$ generators $J_{k}^{\alpha}$ at each site $k$ (normalized so that $\operatorname{tr}\left(J_{k}^{\alpha} J_{k}^{\beta}\right)=\frac{1}{2} \delta^{\alpha \beta}$ ) as

$$
S_{i j}=\frac{1}{n}+2 \sum_{\alpha=1}^{n^{2}-1} J_{i}^{\alpha} J_{j}^{\alpha} .
$$

Note that for spin $1 / 2$ particles $(n=2)$, we have $\mathbf{S}_{i}=\left(J_{i}^{1}, J_{i}^{2}, J_{i}^{3}\right)$.

The HS chain is naturally related to the scalar Sutherland model of $A_{N}$ type [20,21] and its spin version introduced in Refs. [22-24]. In fact, Polychronakos [25] noted that the complete integrability of the HS chain could be deduced from that of the spin Sutherland model by suitably taking the strong coupling limit (the so-called "freezing trick"). Moreover, the latter author applied this technique to construct an integrable spin chain related to the Calogero (rational) model of $A_{N}$ type [26]. The Hamiltonian of this chain, usually referred to in the literature as the Polychronakos-Frahm (PF) chain, reads

$$
\mathcal{H}_{\mathrm{PF}}=\sum_{i<j}\left(\zeta_{i}-\zeta_{j}\right)^{-2}\left(1-S_{i j}\right),
$$


where the chain sites $\zeta_{i}$ are now the equilibrium positions of $N$ particles in the scalar part of the potential of the Calogero spin model of $A_{N}$ type. In particular, the sites of the PF chain are not equally spaced, unlike those of the HS chain. Indeed, it can be shown that the sites of the PF chain are the zeros of the $N$ th Hermite polynomial [27,28], which satisfy the system of algebraic equations

$$
\zeta_{i}=\sum_{j \neq i} \frac{1}{\zeta_{i}-\zeta_{j}}, \quad i=1, \ldots, N
$$

It turns out that both the HS and the PF chains, featuring long-range position-dependent interactions, can be solved in a more detailed and explicit way than the chains of Heisenberg type, characterized by the short range and position independence of the interactions. For instance, the partition function of the models (2) and (3) can be evaluated in closed form for arbitrary $N$ using the freezing trick [29,30], and its expression is relatively simple in both cases. The spectrum, which consists of a set of integers (consecutive in the case of the PF chain), is highly degenerate due to an underlying Yangian symmetry [31]. Moreover, it has been recently shown that even for moderately large $N$ the density of eigenvalues of the HS chain is Gaussian to a high degree of approximation, and that the density of spacings between consecutive levels follows a simple distribution different from the usual Poisson or Wigner laws [30]. In fact, there is strong evidence that these results also hold for other spin chains of Haldane-Shastry type, as, e.g., the $B C_{N}$ or the supersymmetric versions of the original HS chain $[32,33]$.

In this paper we shall consider a novel type of spin chain, which in some sense is intermediate between the Heisenberg and the Polychronakos-Frahm chains. Its Hamiltonian is obtained from that of the PF chain (3)-(4) by retaining only nearest-neighbors interactions, namely

$$
\mathcal{H}=\sum_{i}\left(\xi_{i}-\xi_{i+1}\right)^{-2}\left(1-S_{i, i+1}\right),
$$

where the sites $\xi_{i}$ are defined by restricting the sum in Eq. (4) to nearest neighbors:

$$
\xi_{i}=\frac{1}{\xi_{i}-\xi_{i-1}}+\frac{1}{\xi_{i}-\xi_{i+1}}, \quad i=1, \ldots, N .
$$

In the previous equations we are identifying $S_{N, N+1}$ with $S_{N 1}, \xi_{N+1}$ with $\xi_{1}$ and $\xi_{0}$ with $\xi_{N}$.

We shall see that the chain (5) possesses several remarkable properties, whose study is the purpose of this paper. In the first place, the spin chain (5) is related along the lines of the freezing trick to the spin dynamical model

$$
H=-\sum_{i} \partial_{x_{i}}^{2}+a^{2} r^{2}+\sum_{i} \frac{2 a^{2}}{\left(x_{i}-x_{i-1}\right)\left(x_{i}-x_{i+1}\right)}+\sum_{i} \frac{2 a}{\left(x_{i}-x_{i+1}\right)^{2}}\left(a-S_{i, i+1}\right),
$$

where $r^{2}=\sum_{i} x_{i}^{2}, a>1 / 2$, and we have identified $x_{0} \equiv x_{N}$ and $x_{N+1} \equiv x_{1}$. We have shown in our recent papers $[34,35]$ that an infinite proper subset of the spectrum of the Hamiltonian (7) can be computed in closed form, so that this model is quasi-exactly solvable (QES) [36-38]. The Hamiltonian (7) is a spin version of the QES scalar model

$$
H_{\mathrm{sc}}=\left.H\right|_{S_{i, i+1} \rightarrow 1}
$$

introduced in [39] by Auberson et al. In particular, it was shown in the latter reference that

$$
\mu=\mathrm{e}^{-\frac{a}{2} r^{2}} \prod_{i}\left|x_{i}-x_{i+1}\right|^{a}
$$


is the ground state function of the model (8), with eigenvalue $E_{0}=N a(2 a+1)$. We shall prove that the sites $\xi_{i}$ of the chain (5) are in fact the coordinates of the unique maximum of $\mu$ in the domain

$$
C=\left\{\mathbf{x} \in \mathbb{R}^{N} \mid x_{1}<\cdots<x_{N}\right\} .
$$

By numerically solving Eq. (6), we shall see that for sufficiently large $N$ the chain sites are normally distributed with zero mean and unit variance. We shall present a simple deduction of this property based on the analysis of the continuous limit of the algebraic system (6) as $N \rightarrow \infty$. As a byproduct, we shall obtain an analytic formula providing a very accurate approximation to the sites' coordinates, valid even for moderately large values of $N$. Another nontrivial consequence of this formula is the fact that for large $N$ the strength of the coupling between the spins $i$ and $i+1$ as a function of their mean coordinate $\left(\xi_{i}+\xi_{i+1}\right) / 2$ also follows the Gaussian law, but with zero mean and variance $1 / 2$.

The spectral properties of the spin chain Hamiltonian (5) are also remarkable. Indeed, by a suitable modification of the freezing trick one can show that $\mathcal{H}$ possesses the eigenvalues $0,1,2$ for arbitrary values of $N$ and $n$, and exactly compute their corresponding eigenstates. Our numerical simulations evidence that these energies are the three lowest ones, and that for spin $1 / 2$ none of the remaining eigenvalues of $\mathcal{H}$ are integers. ${ }^{1}$ For $n>2$, the spectrum of $\mathcal{H}$ also contains the integer eigenvalue 3 , which appears to be not the fourth but the fifth lowest energy. The above properties suggest that the model (5) could be regarded as a quasi-exactly solvable chain, in the sense that only a certain number of eigenvalues and their corresponding eigenvectors of the Hamiltonian $\mathcal{H}$ can be computed in closed form.

We have also studied the distribution of energy levels of the chain (5) for a large number of particles. Since the partition function of this chain is not known, we have performed a numerical calculation of the density of levels using the methods of moments [40,41]. It turns out that, in contrast with the typical behavior of spin chains of Haldane-Shastry type, the distribution of levels clearly deviates from the Gaussian law. As an indication of the accuracy of the approximate level density derived via the moments method, we have compared its mean and variance with the exact values obtained by taking traces of suitable powers of the Hamiltonian. From this discussion it also follows that for large $N$ the mean and variance of the energy behave as $N^{3}$ and $N^{5}$, respectively, just as for the trigonometric chains of HS type in Refs. [30,32].

The paper is organized as follows. In Section 2 we study the distributions of the chain sites and the couplings, comparing the results obtained with those for the PF chain. Section 3, which is the core of the paper, is devoted to the determination of the integer eigenvalues of the chain (5) and their corresponding eigenstates. We also present in this section a detailed example for the case of 5 particles of spin 1, which motivates a number of conjectures on the degeneracy of the integer levels. In Section 4 we use the method of moments to approximately compute the density of levels of the chain $\mathcal{H}$, showing that it follows a Wigner-like law. Finally, in Section 5 we summarize our conclusions and outline possible future developments. For the reader's convenience, in Appendix A we present some background material on the exact eigenfunctions of the spin dynamical model (7) used in Section 3, while in Appendix B we include an overview of the method of moments.

\footnotetext{
1 As a matter of fact, the previous assertion does not hold for the cases $N=3$ (for which the chain (5) reduces to the $\mathrm{PF}$ chain, whose eigenvalues are known to be integers), and $N=4$ (for which our numerical simulations indicate that all the eigenvalues are also integers). Therefore, in the rest of the paper we shall exclude these special cases from our discussion.
} 


\section{The chain sites}

We shall start this section by proving that the sites of the chain (5) are the coordinates of the unique maximum in the domain (10) of the ground state function (9) of the scalar Hamiltonian (8). It is convenient to write the ground state as

$$
\mu=\mathrm{e}^{a \lambda(\mathbf{x})},
$$

where

$$
\lambda(\mathbf{x})=\sum_{i} \log \left|x_{i}-x_{i+1}\right|-\frac{r^{2}}{2}
$$

has the same extrema as $\mu$ and is independent of $a$. Thus the equations (6) defining the chain sites are just the conditions for $\xi=\left(\xi_{1}, \ldots, \xi_{N}\right)$ to be a critical point of $\lambda$. The existence of a maximum of $\lambda$ in $C$ is clear, since it is continuous in $C$ and tends to $-\infty$ both on its boundary and as $r \rightarrow \infty$. Uniqueness follows from the fact that the Hessian of $\lambda$ is negative definite in $C$. Indeed, by Gerschgorin's theorem [42, 15.814], the eigenvalues of the Hessian of $\lambda$ at $\mathbf{x}$ lie in the union of the intervals

$$
\left[\frac{\partial^{2} \lambda}{\partial x_{i}^{2}}-\gamma_{i}, \frac{\partial^{2} \lambda}{\partial x_{i}^{2}}+\gamma_{i}\right], \quad \text { where } \gamma_{i}=\sum_{j \neq i}\left|\frac{\partial^{2} \lambda}{\partial x_{i} \partial x_{j}}\right| \text {. }
$$

Since

$$
\begin{aligned}
& \frac{\partial^{2} \lambda}{\partial x_{i}^{2}}=-1-\left(x_{i}-x_{i+1}\right)^{-2}-\left(x_{i}-x_{i-1}\right)^{-2}, \\
& \frac{\partial^{2} \lambda}{\partial x_{i} \partial x_{i \pm 1}}=\left(x_{i}-x_{i \pm 1}\right)^{-2}, \quad \frac{\partial^{2} \lambda}{\partial x_{i} \partial x_{j}}=0 \quad \text { if } \quad j \neq i, i \pm 1,
\end{aligned}
$$

we have

$$
\frac{\partial^{2} \lambda}{\partial x_{i}^{2}}+\gamma_{i}=-1
$$

and thus all the eigenvalues of the Hessian of $\lambda$ are strictly negative.

Since $\lambda$ and $C$ are invariant under the transformation

$$
x_{i} \mapsto-x_{N-i+1}, \quad i=1, \ldots, N,
$$

and $\lambda$ has a unique maximum in $C$, it follows that

$$
\xi_{i}=-\xi_{N-i+1},
$$

so that the chain sites are symmetric about the origin. In particular, the center of mass of the spins vanishes, i.e.,

$$
\bar{\xi} \equiv \frac{1}{N} \sum_{i} \xi_{i}=0
$$

We have numerically solved equations (6) for the chain sites for up to 250 spins. Before presenting our conclusions, a remark on the configuration of these sites is in order. The attentive reader might have been surprised by our claim that the chain (5) features only nearest-neighbors 


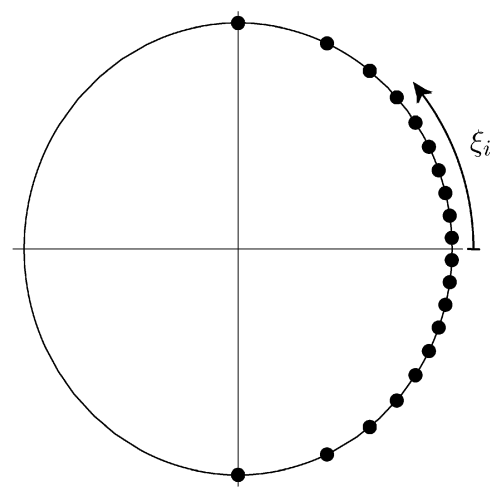

Fig. 1. Sites of the chain $\mathcal{H}$ for $N=20$ spins.

interactions, in spite of the fact that the first spin interacts with the last one. We can avoid this objection by regarding the site coordinate $\xi_{i}$ as an arc length in a circle of radius $2 \xi_{N} / \pi$, see Fig. 1. In this way the spins at the sites $\xi_{1}$ and $\xi_{N}$ are indeed nearest-neighbors and, moreover, the strength of the interactions are inversely proportional to the squared distance between consecutive spins, measured along the arc.

It is apparent from Fig. 1 (and also follows immediately from Eq. (6)) that the sites $\xi_{i}$ are not equally spaced. In fact, our computations show that for large values of $N$ the sites $\xi_{i}$ follow with great accuracy a Gaussian distribution with zero mean and unit variance. More exactly, the cumulative density of sites (normalized to unity)

$$
\mathcal{F}(x)=N^{-1} \sum_{i} \theta\left(x-\xi_{i}\right),
$$

where $\theta$ is Heaviside's step function, is approximately given by

$$
F(x)=\frac{1}{2}[1+\operatorname{erf}(x / \sqrt{2})]
$$

The agreement between the functions $\mathcal{F}$ and $F$ is remarkably good for $N \gtrsim 100$ (see Fig. 2 for the case $N=150$ ) and increases steadily with $N$, e.g., the mean square error of the fit for 100 , 150 and 200 spins are respectively $2.6 \times 10^{-5}, 1.1 \times 10^{-5}$ and $7.9 \times 10^{-6}$.

The fact that for large $N$ the cumulative density of sites is well approximated by the Gaussian law (14) can be justified by the following heuristic argument. Let $x(t, u)$ be a smooth function such that $x(i, N)=\xi_{i}$ for $i=1, \ldots, N$, and define the rescaled function $y(s, \epsilon)=x(s / \epsilon, 1 / \epsilon)$. By Eq. (6), the latter function must satisfy the relation

$$
\frac{1}{y(s, \epsilon)-y(s-\epsilon, \epsilon)}+\frac{1}{y(s, \epsilon)-y(s+\epsilon, \epsilon)}=y(s, \epsilon)
$$

for $\epsilon=1 / N \ll 1$ and $s=\frac{1}{N}, \frac{2}{N}, \ldots, 1$. Let us now assume that Eq. (15) holds for all $s \in \mathbb{R}$ and all $\epsilon \ll 1$. Writing

$$
y(s, \epsilon)=\sum_{k=0}^{\infty} y_{k}(s) \epsilon^{k}
$$




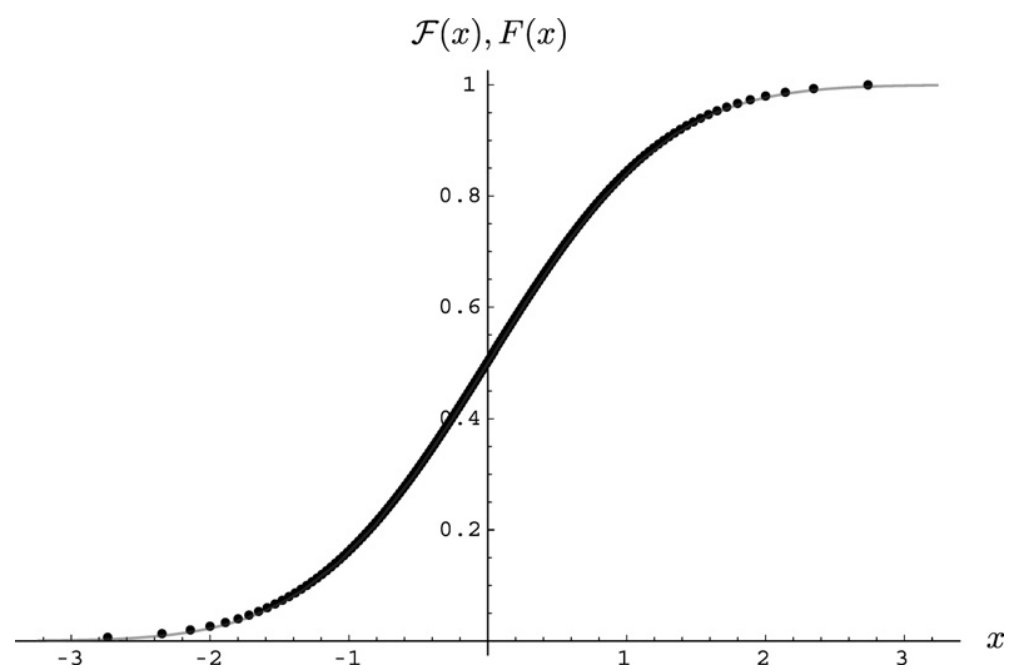

Fig. 2. Cumulative distribution functions $\mathcal{F}(x)$ (at its discontinuity points) and $F(x)$ (continuous grey line) for $N=150$ spins.

and using the expansion

$$
y(s, \epsilon)-y(s \pm \epsilon, \epsilon)=\mp y_{0}^{\prime}(s) \epsilon-\left(\frac{y_{0}^{\prime \prime}(s)}{2} \pm y_{1}^{\prime}(s)\right) \epsilon^{2}+O\left(\epsilon^{3}\right)
$$

the leading term in Eq. (15) yields the differential equation

$$
y_{0}^{\prime \prime}=y_{0} y_{0}^{\prime 2} \text {. }
$$

The general solution of this equation is implicitly given by

$$
s=c_{0}+c_{1} \operatorname{erf}\left(y_{0}(s) / \sqrt{2}\right) .
$$

Hence, up to terms of order $\epsilon=1 / N$, the cumulative distribution function of the chain sites (normalized to unity) is approximated by the continuous function

$$
F(x)=c_{0}+c_{1} \operatorname{erf}(x / \sqrt{2}) .
$$

The normalization conditions $F(-\infty)=0$ and $F(\infty)=1$ imply that $c_{0}=c_{1}=1 / 2$, and thus the empiric law (14) is recovered.

From Eq. (16) (with $c_{0}=c_{1}=1 / 2$ ) it follows that the site $\xi_{k}$ can be determined up to terms of order $1 / N$ by the formula

$$
\xi_{k} \simeq \sqrt{2} \operatorname{erf}^{-1}\left(\frac{2 k-N}{N}\right)
$$

If the sites $\xi_{k}$ were exactly given by the previous formula, they would satisfy the identity

$$
\operatorname{erf}\left(\xi_{k} / \sqrt{2}\right)+\operatorname{erf}\left(\xi_{N-k+1} / \sqrt{2}\right)=\frac{2}{N},
$$

which is clearly inconsistent with the exact relation (11). However, the slightly modified formula

$$
\xi_{k} \simeq \sqrt{2} \operatorname{erf}^{-1}\left(\frac{2 k-N-1}{N}\right)
$$




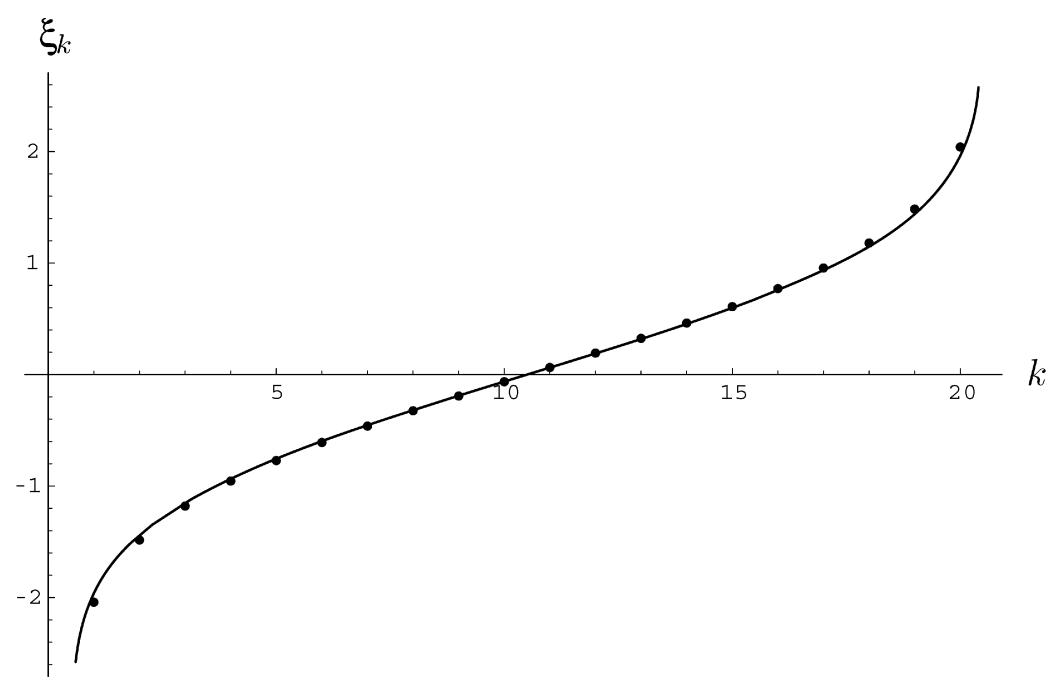

Fig. 3. Sites coordinates $\xi_{k}$ and their continuous approximation (18) for $N=20$ spins.

differs from (17) by a term of order $1 / N$ and is fully consistent with the relation (11). Although both (17) and (18) provide an excellent approximation to the chain sites for large $N$, the latter equation is always more accurate than the former, and can be used to estimate $\xi_{k}$ with remarkable precision even for relatively low values of $N$, cf., Fig. 3.

It is also of interest to determine whether the position of the last spin tends to infinity as $N \rightarrow \infty$, since according to our interpretation of the chain's geometry the number $2 \xi_{N} / \pi$ is the radius of the circle on which the spins lie. From Eq. (18) it follows that for large $N$ the last spin's coordinate $\xi_{N}$ is approximately given by

$$
\xi_{N} \simeq \sqrt{2} \operatorname{erf}^{-1}\left(1-\frac{1}{N}\right),
$$

so that $\xi_{N}$ should diverge as $N \rightarrow \infty$. Of course, this assertion should be taken with some caution, since in Eq. (18) the argument of the inverse error function is correct only up to terms of order $1 / N$. In order to check the correctness of the approximation (19), we use the asymptotic expansion of $\operatorname{erf}^{-1}(u)$ for $u \rightarrow 1$ in Ref. [43] to replace (19) by the simpler formula

$$
\xi_{N} \simeq \sqrt{2 \eta-\log \eta}
$$

where

$$
\eta=\log \left(\frac{N}{\sqrt{\pi}}\right) .
$$

As can be seen in Fig. 4, the approximate formula (20) qualitatively reproduces the growth of $\xi_{N}$ when $N$ ranges from 100 to 250 . A greater accuracy can be achieved by introducing an adjustable parameter in Eq. (19) through the replacement $1 / N \rightarrow \alpha / N$, so that in Eq. (20) $\eta$ becomes

$$
\eta=\log \left(\frac{N}{\alpha \sqrt{\pi}}\right) .
$$

In Fig. 4, we have also plotted the law (20)-(21) with the optimal value $\alpha=0.94$, which is in excellent agreement with the numerical values of $\xi_{N}$ for $N=100,105, \ldots, 250$. 


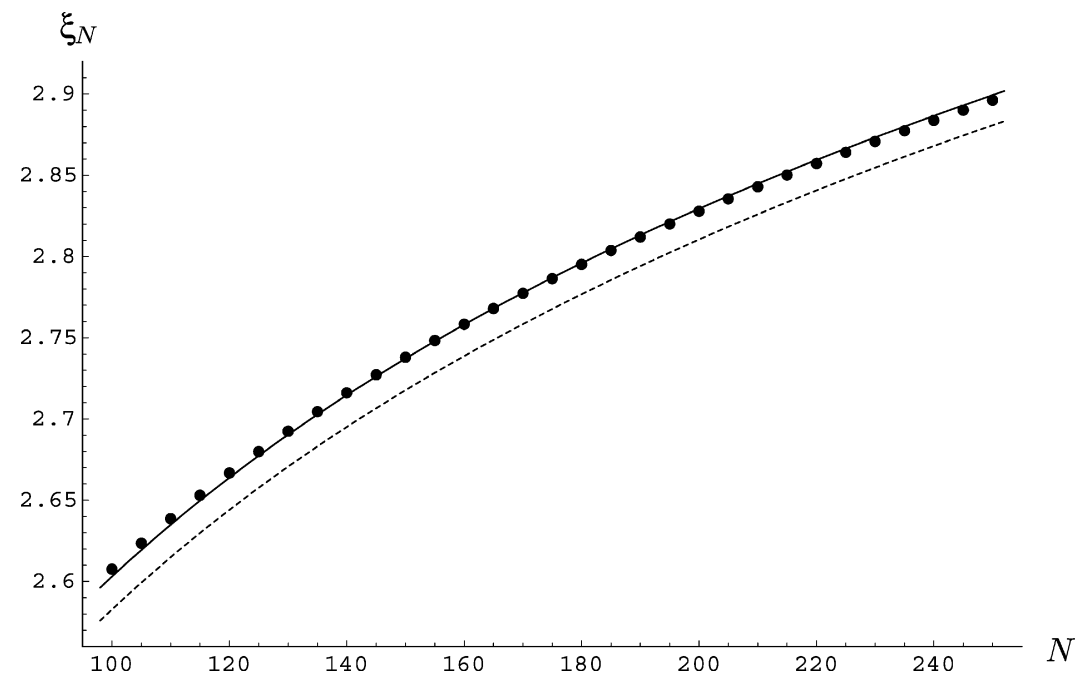

Fig. 4. Position of the last $\operatorname{spin} \xi_{N}$ for $N=100,105, \ldots, 250$ and its continuous approximation (20)-(21) for $\alpha=0.94$ (solid line) and $\alpha=1$ (dashed line).

The last property of the spin chain (5) that we shall analyze in this section is the dependence of the coupling between neighboring spins on their mean coordinate. Calling

$$
h_{k}=\left(\xi_{k}-\xi_{k+1}\right)^{-2}, \quad \bar{\xi}_{k}=\frac{\xi_{k}+\xi_{k+1}}{2},
$$

we shall now see that when $N \gtrsim 100$ the Gaussian law

$$
h_{k} \simeq \frac{N^{2}}{2 \pi} \mathrm{e}^{-\bar{\xi}_{k}^{2}}
$$

holds with remarkable precision, cf. Fig. 5. Indeed, if $x=x(k)$ denotes the RHS of Eq. (18) we have

$$
2 k=N \operatorname{erf}\left(\frac{x}{\sqrt{2}}\right)+N+1
$$

so that

$$
\frac{\mathrm{d} x}{\mathrm{~d} k}=\frac{\sqrt{2 \pi}}{N} \mathrm{e}^{\frac{1}{2} x^{2}}
$$

is of order $1 / N$. Hence, up to terms of order $1 / N$ we have

$$
\left[x\left(k-\frac{1}{2}\right)-x\left(k+\frac{1}{2}\right)\right]^{-2} \simeq\left(\frac{\mathrm{d} x}{\mathrm{~d} k}\right)^{-2}=\frac{N^{2}}{2 \pi} \mathrm{e}^{-x^{2}(k)} .
$$

Since (up to terms of order $1 / N^{2}$ )

$$
x(k) \simeq \frac{1}{2}\left[x\left(k-\frac{1}{2}\right)+x\left(k+\frac{1}{2}\right)\right],
$$

Eq. (23) follows from (25) replacing $k$ by $k+\frac{1}{2}$. 


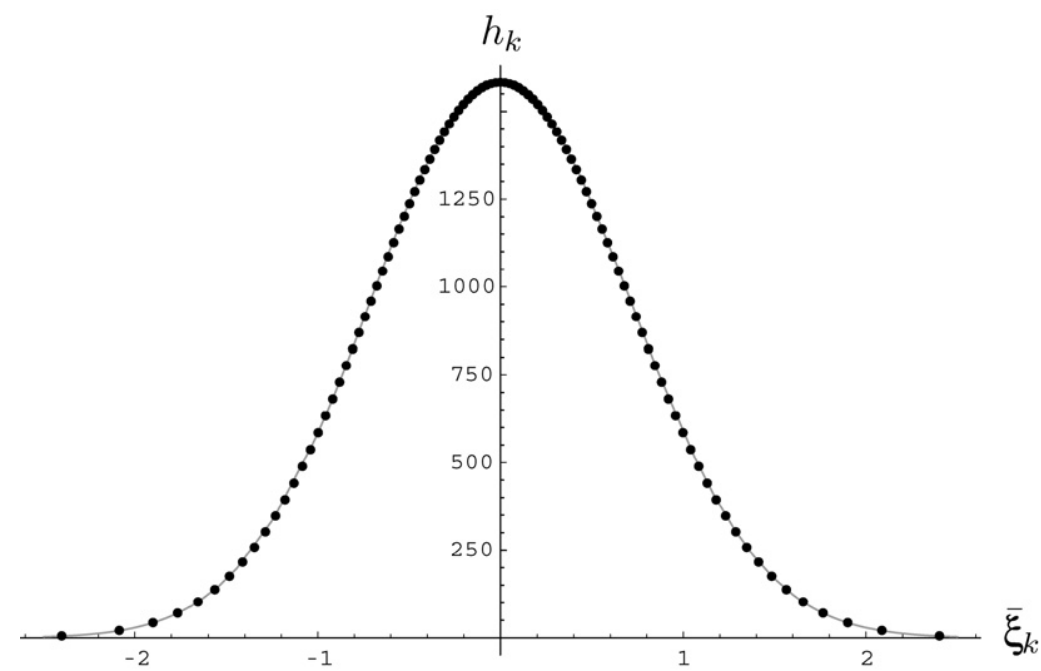

Fig. 5. Plot of the coupling between the spins $k$ and $k+1$ versus their mean position, fitted by the Gaussian (23), for $N=100$ spins.

We shall finish this section with a brief comparison of the previous properties with those of the PF chain (3). For large $N$, the density of sites of the PF chain (normalized to unity), that is the density of zeros of the $N$ th Hermite polynomial, is asymptotically given by the circular law [44]

$$
\rho_{N}(x)=\frac{1}{\pi N} \sqrt{2 N-x^{2}} .
$$

The last site $\zeta_{N}$ of the PF chain grows with $N$ much faster than the corresponding site $\xi_{N}$ of the chain (5), for the largest zero of the $N$ th Hermite polynomial behaves as $\sqrt{2 N}+O\left(N^{-1 / 6}\right)$; see, e.g., [45]. Finally, in contrast with the PF chain, the polynomials determined for each $N \geqslant 2$ by the corresponding sites $\xi_{i}$ of the chain (5) do not form an orthogonal family. In other words, the polynomials

$$
p^{(N)}(z) \equiv \prod_{i}\left(z-\xi_{i}\right)
$$

do not satisfy a three-term recursion relation of the form

$$
p^{(N+1)}(z)=z p^{(N)}(z)-a_{N} p^{(N-1)}(z),
$$

as it can be verified using the explicit expressions

$$
p^{(2)}(z)=z^{2}-1, \quad p^{(3)}(z)=z^{3}-\frac{3 z}{2}, \quad p^{(4)}(z)=z^{4}-2 z^{2}+\frac{1}{4} .
$$

\section{Quasi-exact solvability of the spin chain}

In this section we shall see that the spin chain (5) and the spin dynamical model (7) are related via a slight modification of the usual freezing trick mechanism. We shall exploit this connection to compute in closed form some eigenstates of $\mathcal{H}$ with integer energy, for any number of particles 
and arbitrary spin. We shall then compare our results with those obtained by numerical diagonalization of $\mathcal{H}$ for small values of $N$ and $n$. The numerical computations strongly suggest that the exact states derived in this section exhaust all the eigenstates of the chain (5) with integer energy.

We shall use in what follows the decomposition

$$
H=H_{\mathrm{sc}}+2 a h(\mathbf{x}),
$$

where

$$
h(\mathbf{x})=\sum_{i}\left(x_{i}-x_{i+1}\right)^{-2}\left(1-S_{i, i+1}\right) .
$$

It shall also be convenient to write the scalar Hamiltonian (8) as

$$
H_{\mathrm{sc}}=-\sum_{i} \partial_{x_{i}}^{2}+a^{2} U(\mathbf{x})-a V(\mathbf{x}),
$$

where the scalar potentials $U$ and $V$ are respectively given by

$$
\begin{aligned}
& U(\mathbf{x})=r^{2}+\sum_{i} \frac{2}{\left(x_{i}-x_{i+1}\right)^{2}}+\sum_{i} \frac{2}{\left(x_{i}-x_{i-1}\right)\left(x_{i}-x_{i+1}\right)}, \\
& V(\mathbf{x})=\sum_{i} \frac{2}{\left(x_{i}-x_{i+1}\right)^{2}} .
\end{aligned}
$$

From Eq. (30) it follows that for large $a$ the low-lying eigenfunctions of $H_{\mathrm{sc}}$ concentrate at absolute minima of the potential $U$ in the domain (10) and their energies satisfy $[46,47]$

$$
E=a^{2} \min _{\mathbf{x} \in C} U(\mathbf{x})+O(a) .
$$

By comparison with the exact results for the ground state (9), one concludes that $\boldsymbol{\xi}$ is an absolute minimum of $U$ and $U(\xi)=2 N$. This condition has been used in the previous section to check the accuracy of the numerical solution of the sites equations (6) for large values of $N$.

There are two main limitations which prevent the use of the standard freezing trick argument $[29,32]$ for computing the spectrum of the spin chain (5). The first one is the requirement that $\boldsymbol{\xi}$ be the unique minimum of the potential $U$ in the domain $C$. Although our numerical calculations suggest that this is indeed the case, we have not been able to provide a rigorous proof of this fact. The second limitation, which is more fundamental, is the fact that the dynamical models $H$ and $H_{\mathrm{sc}}$ are only quasi-exactly solvable. Let us briefly recall the basics of the usual freezing trick method in order to understand why the full knowledge of the spectra of $H$ and $H_{\mathrm{sc}}$ is essential for its application. Indeed, if the potential $U$ has an unique minimum $\xi$ in $C$, for sufficiently large $a$ all the eigenfunctions of $H_{\mathrm{sc}}$ are sharply peaked around this point. Thus, if $\psi(\mathbf{x})$ is an eigenfunction of $H_{\mathrm{sc}}$ with energy $E_{\mathrm{sc}}$ and $|\sigma\rangle$ is an eigenstate of the chain $\mathcal{H}$ with eigenvalue $\mathcal{E}$, for $a \gg 1$ we have

$$
h(\mathbf{x}) \psi(\mathbf{x})|\sigma\rangle \simeq \psi(\mathbf{x}) h(\boldsymbol{\xi})|\sigma\rangle \equiv \psi(\mathbf{x}) \mathcal{H}|\sigma\rangle=\mathcal{E} \psi(\mathbf{x})|\sigma\rangle .
$$

By Eq. (28), the state $\psi(\mathbf{x})|\sigma\rangle$ is then an approximate eigenfunction of $H$ with eigenvalue

$$
E \simeq E_{\mathrm{sc}}+2 a \mathcal{E} \text {. }
$$

In other words, the Hamiltonian (7) is approximately diagonal in a basis of the form $\left\{\psi_{i}(\mathbf{x})\left|\sigma_{j}\right\rangle\right\}$, where $\left\{\psi_{i}(\mathbf{x})\right\}$ is a basis of eigenfunctions of $H_{\mathrm{sc}}$ and $\left\{\left|\sigma_{j}\right\rangle\right\}$ is a basis of eigenstates of $\mathcal{H}$. 
Eq. (33) cannot be used directly to compute the corresponding spectrum of $\mathcal{H}$, since it is not clear a priori which pairs of eigenvalues of $H$ and $H_{\mathrm{sc}}$ yield an approximate eigenvalue $\left(E-E_{\mathrm{sc}}\right) /(2 a)$ of $\mathcal{H}$. However, using Eq. (33) one can easily express the partition function $\mathcal{Z}$ of the spin chain $\mathcal{H}$ in terms of the partition functions $Z$ and $Z_{\mathrm{sc}}$ of $H$ and $H_{\mathrm{sc}}$, respectively, via the formula

$$
\mathcal{Z}(T)=\lim _{a \rightarrow \infty} \frac{Z(2 a T)}{Z_{\mathrm{sc}}(2 a T)} .
$$

The latter formula, which is the key result behind the standard freezing trick approach, cannot be used to compute the spectrum of the spin chain $\mathcal{H}$ unless the whole spectrum of both $H$ and $H_{\mathrm{sc}}$ is known.

In spite of the above limitations, we shall see in this section that it is still possible to compute a number of eigenstates of the spin chain (5) from some of the families of spin eigenfunctions of the model (7) constructed in Ref. [35]. More precisely, we will show that certain linear combinations of these eigenfunctions factorize as the product of the ground state of the scalar model (8) times a spin function, whose limit as $a \rightarrow \infty$ is an eigenstate of the spin chain Hamiltonian $\mathcal{H}$.

Let us begin by introducing some preliminary notation. Let $\Sigma$ be the space of internal degrees of freedom of $N$ particles with $\operatorname{su}(n)$ spin, and denote the elements of its canonical basis by $\left|s_{1} \ldots s_{N}\right\rangle$, where $s_{i}=-M,-M+1, \ldots, M$ and $M=(n-1) / 2$. Let $\Lambda$ be the total symmetrizer under particle permutations, that is

$$
\Lambda=\frac{1}{N !} \sum_{k=1}^{N !} \Pi_{k},
$$

where $\Pi_{k}$ denotes a permutation operator acting simultaneously on spatial coordinates and spins. We shall consider in what follows the subspace $\Sigma^{\prime} \subset \Sigma$ of spin states $|s\rangle$ such that $\sum_{i}\left|s_{i, i+1}\right\rangle$ is symmetric, where $\left|s_{i j}\right\rangle$ is defined by

$$
\Lambda\left(x_{1} x_{2}|s\rangle\right)=\sum_{i<j} x_{i} x_{j}\left|s_{i j}\right\rangle ;
$$

see Ref. [35] for a complete characterization of this subspace. Finally, given a state $|s\rangle \in \Sigma$ we define the spin functions

$$
\begin{aligned}
& \Phi^{(k)}(\mathbf{x} ;|s\rangle)=\Lambda\left(x_{1}^{k}|s\rangle\right) \quad(k=0,1,2), \quad \tilde{\Phi}^{(2)}(\mathbf{x} ;|s\rangle)=\Lambda\left(x_{1} x_{2}|s\rangle\right), \\
& \hat{\Phi}^{(3)}(\mathbf{x} ;|s\rangle)=\Lambda\left(x_{1} x_{2}\left(x_{1}-x_{2}\right)|s\rangle\right) .
\end{aligned}
$$

We shall suppress one or both of the arguments of the above spin functions when appropriate.

We shall next present the basic result we have used to construct eigenstates of the chain (5) out of the eigenfunctions of the dynamical spin model (7) in Appendix A. Recall that the energies of these eigenfunctions are the numbers $E_{l m}=E_{0}+2 a(2 l+m)$, where $l$ and $m$ are non-negative integers and $E_{0}$ is the ground state energy of the scalar model $H_{\mathrm{sc}}$.

The key point in the ensuing argument is the fact that for large $a$ the normalized ground state $\mu_{0}=\mu /\|\mu\|$ of $H_{\mathrm{sc}}$, where $\mu$ is given by (9) and $\|\mu\|^{2}=\int_{C} \mu^{2}$, is sharply peaked around its maximum $\xi$. Hence, if $F(\mathbf{x})$ is a continuous spin-dependent function such that the integral of $\mu_{0}^{2} F$ over $C$ is finite, the main contribution to this integral comes from a small ball centered at $\xi$, up to exponentially small terms. By the standard argument behind the proof of Laplace's method, it follows that

$$
\lim _{a \rightarrow \infty} \int_{C} \mu_{0}^{2} F=F(\xi) \lim _{a \rightarrow \infty} \int_{C} \mu_{0}^{2}=F(\xi) .
$$


Assume now that $\Psi(\mathbf{x} ; a)=\mu_{0}(\mathbf{x} ; a) \Phi(\mathbf{x} ; a)$ is a continuous eigenfunction of $H$ with energy $E_{l m}$ such that $\Phi(\mathbf{x} ; a)=\sum_{i=0}^{k} a^{-i} \Phi_{i}(\mathbf{x})$, with each $\Phi_{i}$ a polynomial in $\mathbf{x}$. Denoting by

$$
(f, F)=\int_{C} \overline{f(\mathbf{x})} F(\mathbf{x}) \mathrm{d}^{N} \mathbf{x}
$$

the usual spin-valued inner product of a scalar function $f(\mathbf{x})$ with a spin-valued function $F(\mathbf{x})$, from Eq. (28) we obtain

$$
E_{l m}\left(\mu_{0}^{2}, \Phi\right)=E_{l m}\left(\mu_{0}, \Psi\right)=\left(\mu_{0}, H \Psi\right)=\left(\mu_{0}, H_{\mathrm{sc}} \Psi\right)+2 a\left(\mu_{0}, h \Psi\right) .
$$

Since $H_{\mathrm{sc}}$ is self-adjoint and $h$ is a matrix multiplication operator, the RHS of the previous equation equals

$$
\left(H_{\mathrm{sc}} \mu_{0}, \mu_{0} \Phi\right)+2 a\left(\mu_{0}^{2}, h \Phi\right)=E_{0}\left(\mu_{0}, \mu_{0} \Phi\right)+2 a\left(\mu_{0}^{2}, h \Phi\right)=\left(\mu_{0}^{2},\left(E_{0}+2 a h\right) \Phi\right) .
$$

From Eq. (39) it follows that

$$
0=\left(\mu_{0}^{2},(h-2 l-m) \Phi\right)=\sum_{i=0}^{k} a^{-i}\left(\mu_{0}^{2},(h-2 l-m) \Phi_{i}\right) .
$$

Taking the limit $a \rightarrow \infty$, and using Eq. (38) and the fact that $\mathcal{H}=h(\boldsymbol{\xi})$, we finally obtain

$$
(\mathcal{H}-2 l-m) \Phi_{0}(\xi)=0 .
$$

Thus

$$
\Phi_{0}(\xi)=\lim _{a \rightarrow \infty} \Phi(\xi ; a)
$$

is either zero or an eigenstate of the chain (5) with integer energy $2 l+m$.

We shall now see that the application of the previous method to the eigenfunctions (A.1)(A.5) of the dynamical model (7) listed in Appendix A yields the following types of eigenstates of the chain (5):

$$
\begin{aligned}
& \varphi_{0}(|s\rangle)=\Phi^{(0)}(|s\rangle) \equiv \Lambda|s\rangle, \\
& \varphi_{1}(|s\rangle)=\Phi^{(1)}(\xi ;|s\rangle), \\
& \varphi_{2}(|s\rangle)=\Phi^{(2)}(\xi ;|s\rangle)+(N-1) \tilde{\Phi}^{(2)}(\xi ;|s\rangle), \\
& \varphi_{3}(|s\rangle)=\hat{\Phi}^{(3)}(\xi ;|s\rangle)+2 \Phi^{(1)}(\xi ;|s\rangle) .
\end{aligned}
$$

For the states (42c) $|s\rangle$ must belong to the subspace $\Sigma^{\prime}$ and be symmetric in the first two spins, whereas for the states of type (42d) $|s\rangle$ should be antisymmetric under $S_{12}$. In all cases, the energy of the states $\varphi_{k}(|s\rangle)$ is the integer $k$. We shall see in what follows that the states (42d) of energy 3 only appear for $\operatorname{spin} M>1 / 2(n>2)$.

Note, first of all, that Eq. (A.8) implies that $\xi^{2}=N$, since otherwise each eigenfunction $\Psi_{l 0}^{(0)}$ (cf. Eq. (A.1)) would yield an eigenstate of the chain (5) with eigenvalue $2 l$ for arbitrary $l$. (The fact that $\xi^{2}=N$ can also be established directly from Eq. (6) using the identity $U(\xi)=2 N$ for the scalar potential (31).) Hence we need only consider the eigenfunctions (A.1)-(A.5) with $l=0$. Moreover, from Eqs. (A.1)-(A.5) and (A.9) it follows that the eigenfunctions $\Psi_{0 m}^{(0)}, \Psi_{0 m}^{(2)}$ and $\tilde{\Psi}_{0 m}^{(2)}$ (respectively $\Psi_{0 m}^{(1)}$ and $\hat{\Psi}_{0 m}^{(3)}$ ) vanish identically at $\mathbf{x}=\boldsymbol{\xi}$ for odd (respectively even) $m$. 
For the first type of eigenfunctions (A.1), when $m$ is even Eq. (A.10) implies that

$$
\Phi_{0 m}^{(0)}(\mathbf{x}) \equiv a^{-\frac{m}{2}} \mu_{0}^{-1} \Psi_{0 m}^{(0)} \underset{a \rightarrow \infty}{\longrightarrow} \frac{N^{\frac{m}{2}}}{\left(\frac{m}{2}\right) !} \bar{x}^{m} \Phi^{(0)},
$$

where $\Phi^{(0)}$ is defined in (36). By Eq. (12), the RHS of the previous equation vanishes at $\mathbf{x}=\boldsymbol{\xi}$ unless $m=0$. Thus the eigenfunctions (A.1) only yield the zero energy eigenstates (42a) of $\mathcal{H}$ (when $l=m=0$ ). Note that the fact that any symmetric spin state is an eigenstate of the chain $\mathcal{H}$ with zero energy follows directly from Eq. (5). Conversely, the latter equation implies that the ground state energy of $\mathcal{H}$ is zero and that the corresponding eigenstates are totally symmetric. For this reason we shall concentrate in what follows on the nontrivial states (42b)-(42d).

Let us next examine the eigenfunctions of type (A.2) for $l=0$ and odd $m \geqslant 1$. In this case we have

$$
\Phi_{0 m}^{(1)}(\mathbf{x}) \equiv a^{\frac{1-m}{2}} \mu_{0}^{-1} \Psi_{0 m}^{(1)} \underset{a \rightarrow \infty}{\longrightarrow} \frac{N^{\frac{m-1}{2}}}{\left(\frac{m-1}{2}\right) !} \bar{x}^{m-1}\left(\Phi^{(1)}-\bar{x} \Phi^{(0)}\right),
$$

where $\Phi^{(1)}$ is given by (36). Using again Eq. (12) we conclude that $\lim _{a \rightarrow \infty} \Phi_{0 m}^{(1)}$ vanishes at $\mathbf{x}=\boldsymbol{\xi}$ unless $m=1$. In this case, we have

$$
\lim _{a \rightarrow \infty} \Phi_{01}^{(1)}(\xi)=\Phi^{(1)}(\xi ;|s\rangle) \equiv \varphi_{1}(|s\rangle) .
$$
have

Consider now the eigenfunctions $\Psi_{0 m}^{(2)}$ and $\tilde{\Psi}_{0 m}^{(2)}$ with even $m$. Using again Eq. (A.10) we

$$
\begin{aligned}
& a^{-\frac{m}{2}} \mu_{0}^{-1} \Psi_{0 m}^{(2)}=-\frac{2 N^{\frac{m}{2}} \bar{x}^{m}}{(m-1)\left(\frac{m-2}{2}\right) !} \Phi^{(0)}+O\left(a^{-1}\right), \\
& a^{-\frac{m}{2}} \mu_{0}^{-1} \tilde{\Psi}_{0 m}^{(2)}=\frac{2 N^{\frac{m}{2}} \bar{x}^{m}}{(N-1)(m-1)\left(\frac{m-2}{2}\right) !} \Phi^{(0)}+O\left(a^{-1}\right),
\end{aligned}
$$

where $m \geqslant 2$. Thus the $O(1)$ term of the left-hand sides of Eq. (44) vanish at $\mathbf{x}=\boldsymbol{\xi}$ for all $m \geqslant 2$, on account of Eq. (12). However, if $\Psi_{0 m}^{(2)}$ and $\tilde{\Psi}_{0 m}^{(2)}$ are built from the same spin state $|s\rangle$, the previous equations imply that the $O(1)$ part of the linear combination $a^{-\frac{m}{2}} \mu_{0}^{-1}\left(\Psi_{0 m}^{(2)}+(N-\right.$ 1) $\tilde{\Psi}_{0 m}^{(2)}$ ) vanishes. This observation suggests considering the function

$$
\begin{aligned}
\Phi_{0 m}^{(2)}(\mathbf{x}) & \equiv a^{1-\frac{m}{2}} \mu_{0}^{-1}\left(\Psi_{0 m}^{(2)}+(N-1) \tilde{\Psi}_{0 m}^{(2)}\right) \\
& =\frac{N^{\frac{m}{2}-1}}{\left(\frac{m-2}{2}\right) !} \bar{x}^{m-2}\left[\Phi^{(2)}+(N-1) \tilde{\Phi}^{(2)}-N \bar{x}\left(2 \Phi^{(1)}-\bar{x} \Phi^{(0)}\right)\right]+O\left(a^{-1}\right),
\end{aligned}
$$

where $m \geqslant 2$ is even and $\Phi^{(2)}, \tilde{\Phi}^{(2)}$ are given in Eq. (36). Note that $\Phi_{0 m}^{(2)}$ is only defined for states $|s\rangle \in \Sigma^{\prime}$ symmetric under $S_{12}$, since otherwise $\tilde{\Psi}_{0 m}^{(2)}$ would not be defined. The limit of $\Phi_{0 m}^{(2)}$ as $a \rightarrow \infty$ vanishes at $\mathbf{x}=\boldsymbol{\xi}$ unless $m=2$, in which case we obtain the eigenstate with energy 2

$$
\lim _{a \rightarrow \infty} \Phi_{02}^{(2)}(\boldsymbol{\xi})=\Phi^{(2)}(\xi ;|s\rangle)+(N-1) \tilde{\Phi}^{(2)}(\xi ;|s\rangle) \equiv \varphi_{2}(|s\rangle)
$$

Let us finally turn to the last type of eigenfunctions (A.5) with $l=0$ and odd $m \geqslant 3$. From Eq. (A.10) it immediately follows that

$$
a^{\frac{1-m}{2}} \mu_{0}^{-1} \hat{\Psi}_{0 m}^{(3)}=\frac{4 N^{\frac{m-1}{2}} \bar{x}^{m-1}}{(m-2)\left(\frac{m-3}{2}\right) !}\left(\Phi^{(1)}-\frac{\bar{x}}{m} \Phi^{(0)}\right)+O\left(a^{-1}\right),
$$


whose limit as $a \rightarrow \infty$ vanishes identically at $\mathbf{x}=\boldsymbol{\xi}$ since $m \geqslant 3$ in this case. However, as for the previous states, one can cancel the leading term in $a$ of $\hat{\Psi}_{0 m}^{(3)}$ with a suitable linear combination of $\Psi_{0 m}^{(0)}$ and $\Psi_{0 m}^{(1)}$ all built from the same spin state (necessarily antisymmetric under $S_{12}$ for $\hat{\Psi}_{0 m}^{(3)}$ to be defined). Indeed, using Eqs. (43) and (45) and taking into account that for odd $m$

$$
a^{\frac{1-m}{2}} \mu_{0}^{-1} \Psi_{0 m}^{(0)}=\frac{N^{\frac{m-1}{2}}}{\left(\frac{m-1}{2}\right) !} \bar{x}^{m} \Phi^{(0)}+O\left(a^{-1}\right),
$$

one can easily check that

$$
\Phi_{0 m}^{(3)}(\mathbf{x}) \equiv a^{\frac{3-m}{2}} \mu_{0}^{-1}\left(\hat{\Psi}_{0 m}^{(3)}-\frac{2(m-1)}{m-2} \Psi_{0 m}^{(1)}-\frac{2(m-1)^{2}}{m(m-2)} \Psi_{0 m}^{(0)}\right)=O(1) .
$$

We shall next see that the $O(1)$ term of $\Phi_{0 m}^{(3)}(\mathbf{x})$ vanishes at $\mathbf{x}=\boldsymbol{\xi}$ unless $m=3$. Indeed, Eq. (A.9) implies that for $k^{\prime}>2 k$ the term $\bar{x}^{k^{\prime}} P_{k}^{(\alpha+i, \beta)}(t)$ vanishes at $\mathbf{x}=\boldsymbol{\xi}$ to all orders in $a$. Hence the $O(1)$ terms of $\Phi_{0 m}^{(3)}(\mathbf{x})$ and

$$
a^{\frac{3-m}{2} \bar{x}^{m-3}}\left(P_{\frac{m-3}{2}}^{(\alpha+3, \beta)}(t) \hat{\Phi}^{(3)}-2 \frac{m-1}{m-2} \bar{x}^{2} P_{\frac{m-1}{2}}^{(\alpha+1, \beta)}(t) \Phi^{(1)}\right)
$$

coincide at $\mathbf{x}=\boldsymbol{\xi}$. From Eq. (A.9) it is straightforward to show that the $O(1)$ term of (47) is a linear combination of $\bar{x}^{m-3}$ and $\bar{x}^{m-1}$, thus establishing our claim.

By the previous remarks, we need only compute the $O(1)$ term of (47) for $m=3$, which by Eq. (A.10) is given by

$$
\hat{\Phi}^{(3)}+\frac{2}{N}\left(r^{2}-N(N+2) \bar{x}^{2}\right) \Phi^{(1)} \text {. }
$$

Using Eq. (12) and the identity $\xi^{2}=N$ we finally obtain the eigenstates (42d) of energy 3 :

$$
\lim _{a \rightarrow \infty} \Phi_{03}^{(3)}(\xi)=\hat{\Phi}^{(3)}(\xi ;|s\rangle)+2 \Phi^{(1)}(\xi ;|s\rangle) \equiv \varphi_{3}(|s\rangle) .
$$

The previous discussion guarantees that the nontrivial states $\varphi_{k}(|s\rangle)$ given by Eqs. (42b)-(42d) are eigenstates of the chain $\mathcal{H}$ with energy $k$ provided that they do not vanish. For instance, the states (42b) are easily seen to vanish when the spin state $|s\rangle$ is symmetric, since in this case

$$
\varphi_{1}(|s\rangle)=\Phi^{(1)}(\xi ;|s\rangle)=\bar{\xi}|s\rangle=0 .
$$

This is also the case for the states (42c). Indeed, if $|s\rangle$ is symmetric it clearly belongs to $\Sigma^{\prime}$ and we have

$$
\Phi^{(2)}(\mathbf{x} ;|s\rangle)+(N-1) \tilde{\Phi}^{(2)}(\mathbf{x} ;|s\rangle)=\frac{r^{2}}{N}|s\rangle+\frac{2}{N} \sum_{i<j} x_{i} x_{j}|s\rangle=N \bar{x}^{2}|s\rangle .
$$

Since $\varphi_{2}(|s\rangle)$ coincides with the LHS of this expression evaluated at $\mathbf{x}=\boldsymbol{\xi}$, it vanishes on account of Eq. (12). Less trivially, let $|s\rangle$ be a linear combination of basic states $|\mathbf{s}\rangle \equiv\left|s_{1}, \ldots, s_{N}\right\rangle$ such that $|\mathbf{s}| \leqslant 2$, where $|\mathbf{s}|$ denotes the number of distinct components of $\mathbf{s} \equiv\left(s_{1}, \ldots, s_{N}\right)$. If, in addition, $|s\rangle$ is antisymmetric under $S_{12}$, we shall prove below that

$$
\hat{\Phi}^{(3)}+2\left(\frac{r^{2}}{N} \Phi^{(1)}-\bar{x} \Phi^{(2)}\right)=0,
$$


which implies that $\varphi_{3}(|s\rangle)=0$ by Eq. (12) and the identity $\xi^{2}=N$. In summary, the state $|s\rangle$ in the definition of the states (42b)-(42d) can be taken without loss of generality as follows:

$$
\begin{array}{llrl}
\varphi_{1}(|s\rangle): & |s\rangle \in \Sigma-\Lambda(\Sigma), & \\
\varphi_{2}(|s\rangle): & |s\rangle \in \Sigma^{\prime}-\Lambda(\Sigma), & S_{12}|s\rangle=|s\rangle, \\
\varphi_{3}(|s\rangle): & |s\rangle=\sum_{|\mathbf{s}| \geqslant 3} c_{\mathbf{s}}|\mathbf{s}\rangle, & S_{12}|s\rangle=-|s\rangle .
\end{array}
$$

Note, in particular, that the states (42d) do not appear for spin $1 / 2$ in view of the first condition (51).

Our next task is to study the number of linearly independent states of the form (42b)-(42d) with $|s\rangle$ satisfying the above conditions. The main difficulty in this respect is the fact that the eigenstates $\varphi_{k}(|s\rangle)$ constructed from a set of linearly independent states $|s\rangle$ satisfying conditions (49)-(51) need not be independent. In order to address this problem, it is convenient to introduce some additional notation. Given a basic state $|\mathbf{s}\rangle$ with $|\mathbf{s}|=p$, we define its spin content as the set of pairs $\left(s^{i}, v_{i}\right)$, where $s^{1}<\cdots<s^{p}$ are the distinct components of $\mathbf{s}$ and $v_{i}$ is the number of times that $s^{i}$ appears in $\mathbf{s}$. For instance, the spin content of the basic state $|\mathbf{s}\rangle=|0,-2,1,-2,1\rangle$ is $\{(-2,2),(0,1),(1,2)\}$. We shall say that an arbitrary spin state $|s\rangle$ has well-defined spin content if it is a linear combination of basic states having the same spin content. It is obvious that a set of states whose spin contents are all different is linearly independent. Note also that if $|s\rangle$ has a well-defined spin content, then $\varphi_{k}(|s\rangle)$ has the same spin content. Therefore, it suffices to determine the number of linearly independent states $\varphi_{k}(|s\rangle)$ built from states $|s\rangle$ with welldefined spin content. Clearly, for a given spin content $\left\{\left(s^{i}, v_{i}\right)\right\}$ this number is independent of the particular values of the spin coordinates $s^{i}$. We shall therefore denote by $d_{k}\left(v_{1}, \ldots, v_{p}\right) \equiv d_{k}(\boldsymbol{v})$ the dimension of the linear space of states $\varphi_{k}(|s\rangle)$ with a given spin content $\left\{\left(s^{i}, v_{i}\right)\right\}$. We shall next prove the following upper bounds on these dimensions:

$$
d_{1}(\boldsymbol{v}) \leqslant p-1, \quad d_{2}(\boldsymbol{v}) \leqslant p-1, \quad d_{3}(\boldsymbol{v}) \leqslant\left(\begin{array}{c}
p-1 \\
2
\end{array}\right) .
$$

Consider in the first place the states of the form $\varphi_{1}(|s\rangle)$ with a given spin content. If $|\mathbf{s}\rangle$ and $\left|\mathbf{s}^{\prime}\right\rangle$ are two basic states with the same spin content and differing by a permutation of the last $N-1$ spin coordinates, then $\Lambda\left(x_{1}|\mathbf{s}\rangle\right)=\Lambda\left(x_{1}\left|\mathbf{s}^{\prime}\right\rangle\right)$, which implies that $\varphi_{1}(|\mathbf{s}\rangle)=\varphi_{1}\left(\left|\mathbf{s}^{\prime}\right\rangle\right)$ by Eqs. (36) and (42b). Hence, the space of states $\varphi_{1}(|s\rangle)$ with spin content $\left\{\left(s^{1}, v_{1}\right), \ldots,\left(s^{p}, v_{p}\right)\right\}$ is spanned by the states

$$
\varphi_{1}\left(\left|s^{i} \ldots\right|\right), \quad i=1, \ldots, p,
$$

where the ellipsis denotes any ordering of the remaining $N-1$ spin components corresponding to the above spin content. On the other hand, the $p$ states (53) satisfy the linear relation

$$
\sum_{i=1}^{p} v_{i} \varphi_{1}\left(\left|s^{i} \ldots\right|\right)=0,
$$

which implies the first inequality in (52). Indeed, if $|\mathbf{s}\rangle$ is a basic state with spin content $\left\{\left(s^{i}, v_{i}\right)\right\}$, we have

$$
N \bar{x} \Lambda|\mathbf{s}\rangle=\sum_{i=1}^{N} \Lambda\left(x_{i}|\mathbf{s}\rangle\right)=\sum_{i=1}^{p} v_{i} \Lambda\left(x_{1}\left|s^{i} \ldots\right\rangle\right) .
$$


Setting $\mathbf{x}=\boldsymbol{\xi}$ and using (12) we immediately obtain (54).

Let us turn now to the states of the form $\varphi_{2}(|s\rangle)$, where $|s\rangle$ satisfies (50) and has a well-defined spin content $\left\{\left(s^{1}, v_{1}\right), \ldots,\left(s^{p}, v_{p}\right)\right\}$. The results of our previous paper [35] (see Proposition 4) imply that the space of states of this form is spanned by

$$
\varphi_{2}\left(\left|\chi_{i}\right\rangle\right), \quad i=1, \ldots, p,
$$

where

$$
\left|\chi_{i}\right\rangle=\left\{\begin{array}{l}
v_{i}\left(v_{i}-1\right)\left|s^{i} s^{i} \ldots\right\rangle-\sum_{\substack{1 \leqslant j, k \leqslant p \\
j, k \neq i}} v_{j}\left(v_{k}-\delta_{j k}\right)\left|s^{j} s^{k} \ldots\right\rangle, \quad v_{i}>1, \\
\sum_{\substack{1 \leqslant j \leqslant p \\
j \neq i}} v_{j}\left(\left|s^{i} s^{j} \ldots\right\rangle+\left|s^{j} s^{i} \ldots\right|\right), \quad v_{i}=1 .
\end{array}\right.
$$

However, from [35, Proposition 3] it follows that the $p$ states (55) satisfy the linear relation

$$
\sum_{i=1}^{p} \varphi_{2}\left(\left|\chi_{i}\right\rangle\right)=0
$$

which yields the second inequality in (52).

Consider finally the states of the form $\varphi_{3}(|s\rangle)$, where $|s\rangle$ is antisymmetric under $S_{12}$ and has a certain spin content $\left\{\left(s^{1}, v_{1}\right), \ldots,\left(s^{p}, v_{p}\right)\right\}$. Clearly, the space of such states is spanned by

$$
\varphi_{3}\left(\left|s^{i} s^{j} \ldots\right\rangle-\left|s^{j} s^{i} \ldots\right|\right), \quad 1 \leqslant i<j \leqslant p .
$$

We shall prove below that the latter $\left(\begin{array}{l}p \\ 2\end{array}\right)$ states satisfy the linear relations

$$
\sum_{j=1}^{p} v_{j} \varphi_{3}\left(\left|s^{i} s^{j} \ldots\right\rangle-\left|s^{j} s^{i} \ldots\right|\right)=0, \quad i=1, \ldots, p .
$$

Multiplying the LHS of the $i$ th relation by $v_{i}$ and summing over $i$ we obtain

$$
\sum_{i, j=1}^{p} v_{i} v_{j} \varphi_{3}\left(\left|s^{i} s^{j} \ldots\right\rangle-\left|s^{j} s^{i} \ldots\right|\right),
$$

which vanishes by antisymmetry. Thus we can safely drop one of the identities (59), say the last one, and the first $p-1$ are independent since they can be solved for $\varphi_{3}\left(\left|s^{i} s^{p} \ldots\right\rangle-\left|s^{p} s^{i} \ldots\right\rangle\right)$ for $i=1, \ldots, p-1$. Hence there are at most $\left(\begin{array}{c}p \\ 2\end{array}\right)-(p-1)=\left(\begin{array}{c}p-1 \\ 2\end{array}\right)$ independent states of the form (58), which establishes the last inequality in (52).

We still have to prove the identities (59). To this end, denote by $\Phi_{3} \equiv \Phi_{3}(\mathbf{x} ;|s\rangle)$ the LHS of Eq. (48). Since $\Phi_{3}(\xi ;|s\rangle)=\varphi_{3}(|s\rangle)$, Eq. (59) follow directly from the identities

$$
\sum_{j=1}^{p} v_{j} \Phi_{3}\left(\mathbf{x} ;\left|s^{i} s^{j} \ldots\right\rangle-\left|s^{j} s^{i} \ldots\right|\right)=0, \quad i=1, \ldots, p .
$$


Table 1

Energies and degeneracies of the first six and the last levels of the chain (5) with $N=5$ and $M=1$

\begin{tabular}{lc}
\hline Energy & Degeneracy \\
\hline 0 & 21 \\
1 & 24 \\
2 & 24 \\
2.035 & 15 \\
3 & 6 \\
3.953 & 15 \\
$\cdots$ & $\cdots$ \\
15.033 & 3 \\
\hline
\end{tabular}

In order to establish (60), note first that

$$
\begin{aligned}
r^{2} \Phi^{(1)}\left(\left|s^{i} s^{j} \ldots\right|\right)-N \bar{x} \Phi^{(2)}\left(\left|s^{i} s^{j} \ldots\right|\right) & =-\sum_{l=1}^{N} \Lambda\left(x_{1} x_{l}\left(x_{1}-x_{l}\right)\left|s^{i} s^{j} \ldots\right|\right) \\
& =-\sum_{k=1}^{p} v_{k} \hat{\Phi}^{(3)}\left(\left|s^{i} s^{k} \ldots\right|\right),
\end{aligned}
$$

so that

$$
\begin{aligned}
& \frac{N}{2} \sum_{j=1}^{p} v_{j} \Phi_{3}\left(\left|s^{i} s^{j} \ldots\right\rangle-\left|s^{j} s^{i} \ldots\right|\right) \\
& \quad=N \sum_{j=1}^{p} v_{j} \hat{\Phi}^{(3)}\left(\left|s^{i} s^{j} \ldots\right|\right)-\sum_{j, k=1}^{p} v_{j} v_{k} \hat{\Phi}^{(3)}\left(\left|s^{i} s^{k} \ldots\right|\right)+\sum_{j, k=1}^{p} v_{j} v_{k} \hat{\Phi}^{(3)}\left(\left|s^{j} s^{k} \ldots\right|\right) .
\end{aligned}
$$

Since $\sum_{j=1}^{p} v_{j}=N$, the first two terms of the RHS of the previous formula cancel, whereas the last one vanishes by antisymmetry. This concludes the proof of Eq. (60). Note, finally, that the latter equation for $p=2$ easily yields the identity (48).

\subsection{Example}

We shall now discuss in detail the case $N=5$ and spin $M=1$. The sites of this chain are given by $\xi_{5}=-\xi_{1}=1.469, \xi_{4}=-\xi_{2}=0.584, \xi_{3}=0$. The Hamiltonian (5) is represented by a $243 \times$ 243 real symmetric matrix, whose numerical diagonalization is straightforward. The spectrum consists of 21 different levels, the only integer energies being $0,1,2,3$. For conciseness' sake, we only present the first six levels and the last one with their corresponding degeneracies, cf. Table 1. Notice, in particular, the appearance of a noninteger energy between the third and the fifth levels. The degeneracy of the ground level is given by the number of independent symmetric states, that is, the number of combinations with repetitions of 5 elements from 3 . Turning to the other integer energies, we shall next present a basis of eigenstates of each nontrivial type (42b)(42d). By the previous discussion, we must first determine all the possible spin contents $\left\{\left(s^{i}, v_{i}\right)\right\}$ compatible with conditions (49)-(51), and then construct a basis of states $\varphi_{k}(|s\rangle)$ with each of these spin contents. In practice, the former task is performed in two steps, namely, one first finds all possible degeneracy vectors $\boldsymbol{v}$, and then one determines the spin contents associated with each of these vectors. 
Table 2

List of basic states $|\mathbf{s}\rangle$ generating the states (42b) of unit energy for each degeneracy vector $\boldsymbol{v}$

\begin{tabular}{ll}
\hline $\boldsymbol{v}$ & $|\mathbf{s}\rangle$ \\
\hline$(4,1)$ & $|----0\rangle,|----+\rangle,|0000+\rangle$ \\
$(3,2)$ & $|---00\rangle,|---++\rangle,|000++\rangle$ \\
$(2,3)$ & $|--000\rangle,|--+++\rangle,|00+++\rangle$ \\
$(1,4)$ & $|-0000\rangle,|-++++\rangle,|0++++\rangle$ \\
$(3,1,1)$ & $|---0+\rangle,|0---+\rangle$ \\
$(2,2,1)$ & $|--00+\rangle,|00--+\rangle$ \\
$(2,1,2)$ & $|--0++\rangle,|0--++\rangle$ \\
$(1,3,1)$ & $|-000+\rangle,|000-+\rangle$ \\
$(1,2,2)$ & $|-00++\rangle,|00-++\rangle$ \\
$(1,1,3)$ & $|-0+++\rangle,|0-+++\rangle$ \\
\hline
\end{tabular}

Table 3

States $|\chi\rangle$ of the form (56) generating the states (55) of energy 2 for each degeneracy vector $v$

\begin{tabular}{ll}
\hline $\boldsymbol{v}$ & $|\chi\rangle$ \\
\hline$(4,1)$ & $|----0\rangle,|----+\rangle,|0000+\rangle$ \\
$(3,2) \quad$ & $3|---00\rangle-|00---\rangle, 3|---++\rangle-|++---\rangle$, \\
& $3|000++\rangle-|++000\rangle$ \\
& $3|000--\rangle-|--000\rangle, 3|+++--\rangle-|--+++\rangle$, \\
& $3|+++00\rangle-|00+++\rangle$ \\
& $|0000-\rangle,|++++-\rangle,|++++0\rangle$ \\
$(1,4)$ & $6|---0+\rangle-|0+---\rangle-|+0---\rangle$, \\
$(3,1,1) \quad$ & $3(|0---+\rangle+|-0--+\rangle)+|0+---\rangle+|+0---\rangle$ \\
& $|--00+\rangle-|00--+\rangle-|0+--0\rangle-|+00--\rangle$, \\
$(2,2,1) \quad$ & $|00--+\rangle-|--00+\rangle-|-+-00\rangle-|+--00\rangle$ \\
& $|--0++\rangle-|++--0\rangle-|0++--\rangle-|+0--+\rangle$, \\
$(2,1,2) \quad$ & $|++--0\rangle-|--++0\rangle-|-0-++\rangle-|0--++\rangle$ \\
& $3(|-000+\rangle+|0-00+\rangle)+|-+000\rangle+|+-000\rangle$, \\
$(1,3,1) \quad$ & $6|000-+\rangle-|-+000\rangle-|+-000\rangle$ \\
& $|00-++\rangle-|++-00\rangle-|-++00\rangle-|+-00+\rangle$, \\
$(1,2,2) \quad$ & $|++-00\rangle-|00-++\rangle-|-00++\rangle-|0-0++\rangle$ \\
& $|-0+++\rangle+|0-+++\rangle+3(|-+++0\rangle+|+-0++\rangle)$, \\
$(1,1,3) \quad 6|+++-0\rangle-|-0+++\rangle-|0-+++\rangle$ \\
\end{tabular}

The states (42b) of unit energy are generated by basic states $|\mathbf{s}\rangle$ with $p \geqslant 2$ different spin components. In Table 2 we present the list of such basic states, where we have taken into account the relation (54) to drop one state for each different spin content; for instance, the state $|0----\rangle$ does not appear in the table, since $\varphi_{1}(|0----\rangle)=-4 \varphi_{1}(|----0\rangle)$. In this way we obtain 24 basic states, whose corresponding states $\varphi_{1}(|\mathbf{s}\rangle)$ turn out to be linearly independent.

Similarly, the space of states $\varphi_{2}(|s\rangle)$ of energy 2 is spanned by the states (55) with $\left|\chi_{i}\right\rangle$ given by (56), for each spin content $\left\{\left(s^{1}, v_{1}\right), \ldots,\left(s^{p}, v_{p}\right)\right\}$ such that $p \geqslant 2$. As in the previous case, the relation (57) implies that for each spin content one can drop one of the states $\left|\chi_{i}\right\rangle$. In Table 3 we present a possible choice of the 24 states $\left|\chi_{i}\right\rangle$ that can be constructed by this procedure. Just as before, the corresponding states $\varphi_{2}\left(\left|\chi_{i}\right\rangle\right)$ are easily seen to be linearly independent.

Finally, for spin 1 the set of states $\varphi_{3}(|s\rangle)$ of energy 3 is spanned by states of the form (58) associated with spin contents with three different components, cf. Eq. (51). By the last inequality 
Table 4

List of states $|s\rangle$ generating the states (42d) of energy 3 and their corresponding degeneracy vectors $v$

\begin{tabular}{ll}
\hline $\boldsymbol{v}$ & $|s\rangle$ \\
\hline$(3,1,1)$ & $|0+---\rangle-|+0---\rangle$ \\
$(2,2,1)$ & $|+--00\rangle-|-+-00\rangle$ \\
$(2,1,2)$ & $|0--++\rangle-|-0-++\rangle$ \\
$(1,3,1)$ & $|-+000\rangle-|+-000\rangle$ \\
$(1,2,2)$ & $|-00++\rangle-|0-0++\rangle$ \\
$(1,1,3)$ & $|-0+++\rangle-|0-+++\rangle$ \\
\hline
\end{tabular}

(52), for each such spin content there is at most one independent state of the form (58). Thus there are at most 6 independent states of the form (42d), generated (for instance) by the states $|s\rangle$ listed in Table 4. As in the previous cases, it can be checked that the 6 states $\varphi_{3}(|s\rangle)$ constructed from the states in Table 4 are actually independent.

Several important remarks can be made in connection with the previous example. In the first place, it is apparent that the inequalities (52) are in this case equalities for every spin content. Secondly, the number of independent states with integer energy $k$ of the form $\varphi_{k}(|s\rangle)$ coincides with the degeneracy of the corresponding level, cf. Table 1. Hence, in this example all the eigenstates with integer energy are of the form (42), and can therefore be computed explicitly. In fact, we have performed a similar study for the case of $N=6$ and spin $M=3 / 2$, arriving at exactly the same conclusions. In view of these examples, it is natural to formulate the following conjectures:

(1) The inequalities (52) are always equalities.

(2) The only integer energies of the chain (5) are 0,1,2 and (for $M \geqslant 1$ ) 3, corresponding to the first three and the fifth levels.

(3) The only eigenstates with integer energy are those of the form (42).

According to the first conjecture, for $k=1,2,3$ the number of independent states $\varphi_{k}(|s\rangle)$ with a well-defined spin content with $p$ elements depends only on $p$, and is given by

$$
d_{k}(p)= \begin{cases}p-1, & k=1,2, \\
\left(\begin{array}{c}
p-1 \\
2
\end{array}\right), & k=3 .\end{cases}
$$

By the third conjecture, the degeneracy of the integer energy $k$ is thus

$$
d_{k}=\sum_{p=1}^{\min (n, N)} d_{k}(p)\left(\begin{array}{l}
n \\
p
\end{array}\right)\left(\begin{array}{c}
N-1 \\
p-1
\end{array}\right), \quad k=1,2,3 .
$$

Indeed, there are $\left(\begin{array}{l}n \\ p\end{array}\right)$ different choices of $p$ distinct spin values $s^{i}$, and $\left(\begin{array}{c}N-1 \\ p-1\end{array}\right)$ ways of selecting $p$ numbers $v_{i} \geqslant 1$ such that $v_{1}+\cdots+v_{p}=N$. In order to evaluate the sum in (62), note first that

$$
\sum_{p=1}^{\min (n, N)}\left(\begin{array}{c}
p-1 \\
j
\end{array}\right)\left(\begin{array}{l}
n \\
p
\end{array}\right)\left(\begin{array}{c}
N-1 \\
p-1
\end{array}\right)=\left(\begin{array}{c}
N-1 \\
j
\end{array}\right) \sum_{p=1}^{\min (n, N)}\left(\begin{array}{l}
n \\
p
\end{array}\right)\left(\begin{array}{c}
N-j-1 \\
p-j-1
\end{array}\right) .
$$

On the other hand, expanding both sides of the identity

$$
\left(1+z^{-1}\right)^{n}(1+z)^{N-j-1}=z^{-n}(1+z)^{N+n-j-1}
$$


in powers of $z$, we easily obtain

$$
\sum_{p=1}^{\min (n, N)}\left(\begin{array}{l}
n \\
p
\end{array}\right)\left(\begin{array}{c}
N-j-1 \\
p-j-1
\end{array}\right)=\left(\begin{array}{c}
N+n-j-1 \\
N
\end{array}\right) .
$$

The previous identity and Eq. (63) immediately yield the following explicit formula for the degeneracy of the positive integer energies of the chain (5):

$$
d_{k}=\left(\begin{array}{c}
N-1 \\
k-1+\delta_{k 1}
\end{array}\right)\left(\begin{array}{c}
N+n-k-\delta_{k 1} \\
N
\end{array}\right), \quad k=1,2,3 .
$$

(The degeneracy of the ground level $\mathcal{E}=0$ is the dimension of the space of symmetric states, namely $\left(\begin{array}{c}N+n-1 \\ N\end{array}\right)$.)

We have numerically diagonalized the chain Hamiltonian (5) for several values of $n$ and $N$ such that $n^{N} \leqslant 3^{8}=6561$. In all cases, we have checked the validity of the second conjecture, and that the formula (64) for the degeneracies of the integer energies (which is a direct consequence of the first and third conjectures) is satisfied. These results lend strong numerical support to the above conjectures, whose rigorous proof deserves further investigation.

\section{Statistical analysis of the spectrum}

It has been recently shown that for large $N$ the level density of spin chains of HS type can be approximated with remarkable accuracy by a Gaussian distribution [30,32,33]. A natural question is whether this is also the case for the spin chain (5) under consideration. The fact that the partition function of the chain (5) has not been computed in closed form-unlike those of the HS chains in the previous references-makes it difficult to address this problem directly. Although one can in principle diagonalize numerically the matrix of the chain Hamiltonian $\mathcal{H}$, in practice this is only feasible for relatively small values of $N$. In this section we shall study the level density of the chain (5) using the method of moments outlined in Appendix B, which provides an accurate estimation of the level density for larger values of $N$.

In order to improve the numerical stability of the method, it is convenient to take the parameters $b$ and $c$ in Eq. (B.11) as

$$
b=0, \quad c=1,
$$

so that the spectrum of $\mathcal{A}$ lies in the interval $[-2,2]$. Therefore the matrix $\mathcal{A}$ and the Hamiltonian $\mathcal{H}$ are related by

$$
\mathcal{A}=\frac{4 \mathcal{H}}{\mathcal{E}_{\max }}-2
$$

where $\mathcal{E}_{\max }$ is the largest eigenvalue of $\mathcal{H}$. Note that this eigenvalue can be computed numerically without difficulty for relatively large values of $N$, since the matrix of $\mathcal{H}$ is very sparse. One must then check that the coefficients $b_{k}$ and $c_{k}$ in the continued fraction expansion of the resolvent of $\mathcal{A}$ (cf. Appendix B) approximately stabilize to the values $b$ and $c$ in Eq. (65) for $k_{0}<k<k_{1}$, with $k_{1} \gg k_{0}$. We have verified that this is indeed the case for $N$ sufficiently large, where $k_{0}$ is typically of the order of 10 . We can thus use Eq. (B.12) to obtain a continuous approximation $g(x)$, where $x \equiv\left(4 \mathcal{E} / \mathcal{E}_{\max }\right)-2$, to the density of eigenvalues of the matrix $\mathcal{A}$, as explained in Appendix B. 


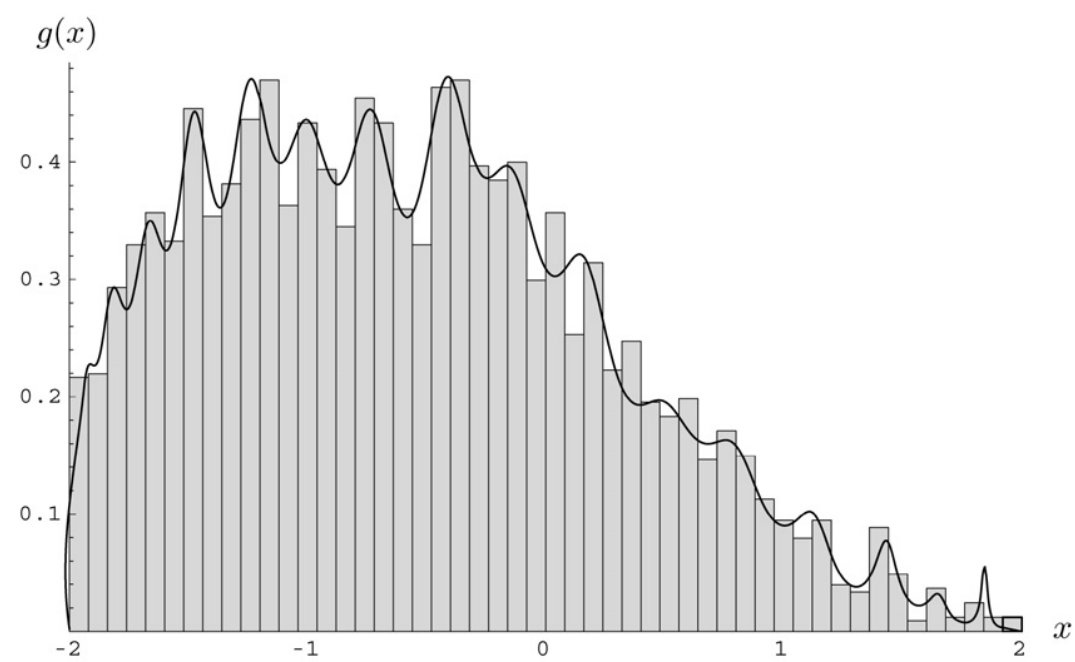

Fig. 6. Continuous approximation $g(x)$ to the density of eigenvalues of the matrix (66) for spin $1 / 2$ and $N=12$ $\left(\mathcal{E}_{\max }=201.21\right)$ compared with the histogram of the spectrum of this matrix.

As a test of the accuracy of the method, we have computed the density $g$ for spin $1 / 2$ and $N=12$, since in this case the matrix $\mathcal{A}$ can still be diagonalized numerically. In Fig. 6 we have compared the approximate density $g$ computed by applying the moments method with $k_{0}=20$ and 20 random vectors with the histogram of the spectrum of $\mathcal{A}$ obtained by subdividing the interval $[-2,2]$ in 50 subintervals. As can be seen from the latter figure, the continuous density $g$ essentially reproduces the shape of the histogram.

The real interest of the method of moments is the possibility of approximating the density of eigenvalues of a large matrix whose numerical diagonalization is not feasible. We have been able to compute the approximate density $g(x)$ of eigenvalues of the matrix $\mathcal{A}$ for $N$ up to 21 for spin $1 / 2$, and up to 13 for spin 1 . For instance, in Fig. 7 we present the plot of $g$ for spin $1 / 2$ and $N=21$, computed with $k_{0}=20$ and 20 random vectors. It is apparent from this plot, and for similar plots for spins $1 / 2$ and 1 , that the level density is not Gaussian. As a matter of fact, the function $g$ is well approximated by the Wigner-like distribution

$$
f(x)=\mathcal{N}^{-1} y^{\alpha} \mathrm{e}^{-\frac{(y-\gamma)^{2}}{\beta}}, \quad y=x+2,
$$

where the normalization constant $\mathcal{N}$ is given by

$$
\mathcal{N}=\frac{1}{2} \alpha \beta^{\frac{\alpha}{2}} \gamma \Gamma\left(\frac{\alpha}{2}\right){ }_{1} F_{1}\left(\frac{1-\alpha}{2}, \frac{3}{2} ;-\frac{\gamma^{2}}{\beta}\right)+\frac{1}{2} \beta^{\frac{\alpha+1}{2}} \Gamma\left(\frac{\alpha+1}{2}\right){ }_{1} F_{1}\left(-\frac{\alpha}{2}, \frac{1}{2} ;-\frac{\gamma^{2}}{\beta}\right),
$$

and ${ }_{1} F_{1}$ is the confluent hypergeometric function of the first kind. Thus the behavior of the chain $\mathcal{H}$ is rather different in this respect from that of the spin chains of HS type.

When $N$ is greater than 12, the accuracy of the level density obtained by the method of moments cannot be gauged directly by computing the spectrum of the matrix $\mathcal{A}$. An indirect way of estimating this accuracy consists in comparing the first few moments of the density $g$ with those of the spectrum of $\mathcal{A}$, computed by taking the traces of appropriate powers of $\mathcal{H}$. As an example, we shall next derive simple expressions for the mean and variance of the spectrum of $\mathcal{H}$ in terms of finite sums involving only the coordinates of the chain sites, which can be easily 


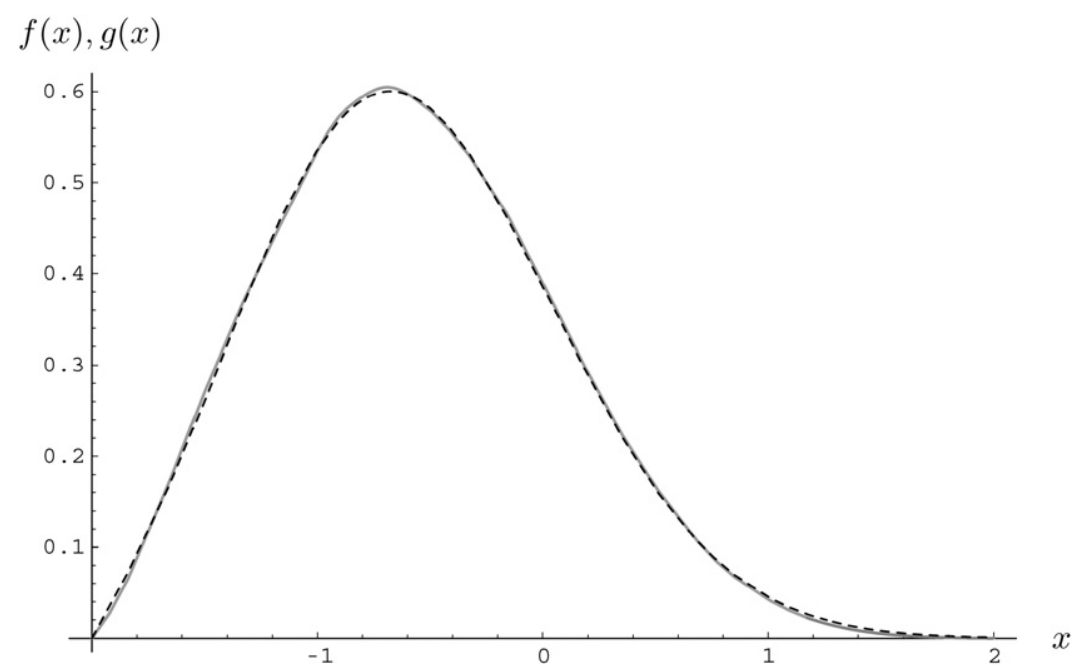

Fig. 7. Approximate density $g(x)$ of eigenvalues of the matrix (66) (grey line) for spin $1 / 2$ and $N=21\left(\mathcal{E}_{\max }=1126.53\right)$, compared with the distribution (67) with optimal parameters $\alpha=0.85, \beta=1.27, \gamma=0.91$ (dashed line).

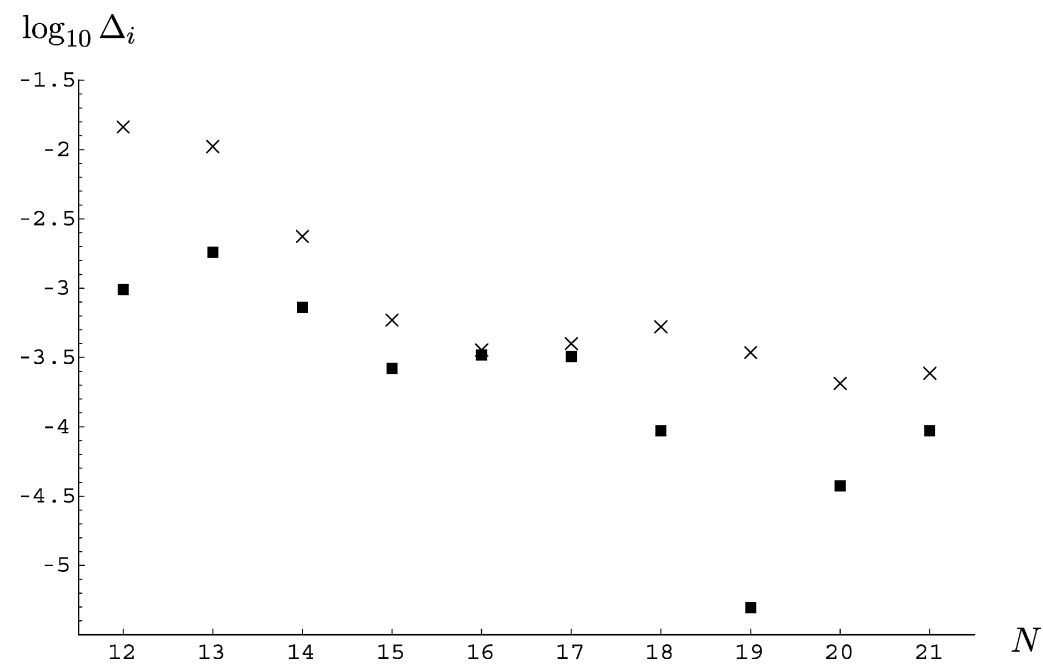

Fig. 8. Logarithmic plot of the relative errors $\Delta_{1}$ (black boxes) and $\Delta_{2}$ (crosses) in Eq. (70) as a function of the number of sites $N$ for spin $1 / 2$. The approximate level density $g$ in Eq. (71) has been computed taking $k_{0}=20$ and averaging over 20 random vectors.

approximated for very large values of $N$. We shall see in this way that the agreement between these values and those obtained from $g$ is indeed very good and, roughly speaking, improves as $N$ grows.

In the first place, the mean energy $\overline{\mathcal{E}}$ of $\mathcal{H}$ can be easily computed noting that $\operatorname{tr} S_{i, i+1}=n^{N-1}$, so that (cf. Eq. (22))

$$
\overline{\mathcal{E}}=n^{-N} \operatorname{tr} \mathcal{H}=n^{-N} \sum_{i} h_{i}\left(n^{N}-n^{N-1}\right)=\left(1-\frac{1}{n}\right) \sum_{i} h_{i}
$$


Similarly, since

$$
\mathcal{H}^{2}=\sum_{i, j} h_{i} h_{j}\left(1-S_{i, i+1}-S_{j, j+1}+S_{i, i+1} S_{j, j+1}\right)
$$

and

$$
\operatorname{tr}\left(S_{i, i+1} S_{j, j+1}\right)=n^{N-2+2 \delta_{i j}},
$$

a straightforward calculation yields

$$
\operatorname{tr}\left(\mathcal{H}^{2}\right)=(n-1) n^{N-2}\left((n-1)\left(\sum_{i} h_{i}\right)^{2}+(n+1) \sum_{i} h_{i}^{2}\right),
$$

so that the variance of the energy is given by

$$
\sigma^{2} \equiv n^{-N} \operatorname{tr}\left(\mathcal{H}^{2}\right)-\overline{\mathcal{E}}^{2}=\left(1-\frac{1}{n^{2}}\right) \sum_{i} h_{i}^{2}
$$

In Fig. 8 we present a logarithmic plot of the relative errors

$$
\Delta_{1} \equiv \frac{\left|\overline{\mathcal{E}}-\overline{\mathcal{E}}_{g}\right|}{\overline{\mathcal{E}}}, \quad \Delta_{2} \equiv \frac{\left|\sigma^{2}-\sigma_{g}^{2}\right|}{\sigma^{2}}
$$

between the exact values (68)-(69) and their approximations

$$
\overline{\mathcal{E}}_{g}=\frac{\mathcal{E}_{\max }}{4} \int_{-2}^{2}(x+2) g(x) \mathrm{d} x, \quad \sigma_{g}^{2}=\frac{\mathcal{E}_{\max }^{2}}{16} \int_{-2}^{2}(x+2)^{2} g(x) \mathrm{d} x-\overline{\mathcal{E}}_{g}^{2}
$$

for $N=12, \ldots, 21$ and spin $1 / 2$. From the latter plot it is apparent that for $N \geqslant 15$ both errors are less than $0.1 \%$, which suggests that for large $N$ the continuous function $g(x)$ computed by the method of moments is indeed an excellent approximation to the level density of the matrix $\mathcal{A}$. From Eqs. (68) and (69) and the discussion of the distribution of the chain sites in Section 2 it is straightforward to deduce the asymptotic behavior of the mean and the variance of the energy for large $N$. Indeed, using Eq. (24) we easily obtain

$$
h_{k} \equiv\left(\xi_{k+1}-\xi_{k}\right)^{-2} \simeq[x(k+1)-x(k)]^{-2} \simeq\left(\frac{\mathrm{d} x}{\mathrm{~d} k}\right)^{-2} \simeq \frac{N^{2}}{2 \pi} \mathrm{e}^{-\xi_{k}^{2}},
$$

so that

$$
\begin{aligned}
\sum_{k} h_{k} & \simeq \frac{N^{2}}{2 \pi} \sum_{k} \mathrm{e}^{-\xi_{k}^{2}} \simeq \frac{N^{2}}{2 \pi} \sum_{k} \mathrm{e}^{-\xi_{k}^{2}} \cdot\left(\xi_{k+1}-\xi_{k}\right) \frac{N}{\sqrt{2 \pi}} \mathrm{e}^{-\frac{\xi_{k}^{2}}{2}} \simeq \frac{N^{3}}{(2 \pi)^{3 / 2}} \int_{-\infty}^{\infty} \mathrm{e}^{-\frac{3}{2} x^{2}} \mathrm{~d} x \\
& =\frac{N^{3}}{2 \pi \sqrt{3}} .
\end{aligned}
$$

Hence for large $N$ the mean energy $\overline{\mathcal{E}}$ behaves as

$$
\overline{\mathcal{E}} \simeq \frac{N^{3}}{2 \pi \sqrt{3}}\left(1-\frac{1}{n}\right) .
$$


Table 5

A comparison of some properties of the spin chain $\mathcal{H}$ versus the Heisenberg chain $\mathcal{H}_{\mathrm{He}}$ and the Polychronakos-Frahm chain $\mathcal{H}_{\mathrm{PF}}$ (the properties followed by an asterisk are based on unpublished work by the authors)

\begin{tabular}{llll}
\hline & $\mathcal{H}$ & $\mathcal{H}_{\mathrm{He}}$ & $\mathcal{H}_{\mathrm{PF}}$ \\
\hline Chain sites distribution & Gaussian & Equispaced & Circular law (26) \\
Solvability & Quasi-exact & Exact & Exact \\
Integer energies & First few & No & All \\
Level density & Wigner-like law (67) & Wigner-like* & Gaussian* \\
Mean energy growth & $N^{3}$ & $N$ & $N^{2^{*}}$ \\
Energy variance growth & $N^{5}$ & $N$ & $N^{3^{*}}$ \\
\hline
\end{tabular}

a Only for spin $1 / 2$.

Similarly,

$$
\sum_{k} h_{k}^{2} \simeq \frac{N^{4}}{4 \pi^{2}} \sum_{k} \mathrm{e}^{-2 \xi_{k}^{2}} \cdot\left(\xi_{k+1}-\xi_{k}\right) \frac{N}{\sqrt{2 \pi}} \mathrm{e}^{-\frac{\xi_{k}^{2}}{2}} \simeq \frac{N^{5}}{(2 \pi)^{5 / 2}} \int_{-\infty}^{\infty} \mathrm{e}^{-\frac{5}{2} x^{2}} \mathrm{~d} x=\frac{N^{5}}{4 \pi^{2} \sqrt{5}},
$$

and therefore the large $N$ limit of the variance of the energy is given by

$$
\sigma^{2} \simeq \frac{N^{5}}{4 \pi^{2} \sqrt{5}}\left(1-\frac{1}{n^{2}}\right) .
$$

Thus, the leading behavior of the energy mean and variance of the chain (5) is analogous to that of the trigonometric HS spin chains studied in Refs. [30,32]. In particular, both quantities grow with $N$ much faster than their counterparts for the Heisenberg chain (both of which diverge as $N)$.

\section{Conclusions}

In this paper we have studied a novel spin chain with position-dependent nearest neighbors interactions, which is intermediate between the Heisenberg chain (position-independent, nearest neighbors interactions) and the spin chains of Haldane-Shastry type (position-dependent, longrange interactions); see Table 5 for a brief comparison.

We have developed a new method related to Polychronakos's freezing trick which has made it possible to compute in closed form a certain number of energy levels with their corresponding eigenstates, for any number of particles and arbitrary spin. While the eigenvalues of the original Haldane-Shastry and Polychronakos-Frahm chains (the closest HS analogs of the chain under consideration) are all integers, the only integer eigenvalues of the new chain are precisely those that have been computed exactly by our method. This fact strongly suggests that the chain (5) should be regarded as the first instance of a quasi-exactly solvable spin chain, thus extending the usual notion of quasi-exact solvability to finite-dimensional Hamiltonians. It is interesting to note, in this respect, that the three lowest frequencies of the small oscillations near the equilibrium of the classical potential (31) are also integers [48].

The method developed in this paper is quite general, and only relies on the explicit knowledge of a number of eigenfunctions of the corresponding spin dynamical model. In this respect, it goes one step beyond the usual freezing trick, which requires the computation in closed form of the whole spectrum of the related dynamical model. In particular, our method is well-suited to spin chains whose associated dynamical model is only quasi-exactly solvable, like for instance the models with elliptic interactions constructed in Refs. [49,50]. 


\section{Acknowledgements}

This work was partially supported by the DGI under grant no. FIS2005-00752, and by the Complutense University and the DGUI under grant No. GR69/06-910556. A.E. acknowledges the financial support of the Spanish Ministry of Education and Science through an FPU scholarship. The authors would also like to thank V. Martín-Mayor for useful discussions on the moments method.

\section{Appendix A. Exact eigenfunctions of the dynamical spin model (7)}

In this appendix we shall list the eigenfunctions of the dynamical spin model (7) used to construct the eigenstates of the chain (5) presented in Section 3. Let

$$
\alpha=N\left(a+\frac{1}{2}\right)-\frac{3}{2}, \quad \beta \equiv \beta(m)=1-m-N\left(a+\frac{1}{2}\right), \quad t=\frac{2 r^{2}}{N \bar{x}^{2}}-1,
$$

where $\bar{x}=\frac{1}{N} \sum_{i} x_{i}$ is the center of mass coordinate. In Ref. [35] it was shown that the model (7) possesses the following families of spin eigenfunctions with energy $E_{l m}=E_{0}+2 a(2 l+m)$, where $l \geqslant 0$ and $m$ is as indicated in each case:

$$
\begin{aligned}
\Psi_{l m}^{(0)}= & \mu_{0} \bar{x}^{m} L_{l}^{-\beta}\left(a r^{2}\right) P_{\left\lfloor\frac{m}{2}\right\rfloor}^{(\alpha, \beta)}(t) \Phi^{(0)}, \quad m \geqslant 0, \\
\Psi_{l m}^{(1)}= & \mu_{0} \bar{x}^{m-1} L_{l}^{-\beta}\left(a r^{2}\right) P_{\left\lfloor\frac{m-1}{2}\right\rfloor}^{(\alpha+1, \beta)}(t)\left(\Phi^{(1)}-\bar{x} \Phi^{(0)}\right), \quad m \geqslant 1, \\
\Psi_{l m}^{(2)}= & \mu_{0} \bar{x}^{m-2} L_{l}^{-\beta}\left(a r^{2}\right)\left[P_{\left\lfloor\frac{m}{2}\right\rfloor-1}^{(\alpha+2, \beta)}(t)\left(\Phi^{(2)}-2 \bar{x} \Phi^{(1)}+\bar{x}^{2} \Phi^{(0)}\right)\right. \\
& \left.-\frac{2(\alpha+1)}{2\left\lfloor\frac{m-1}{2}\right\rfloor+1} \bar{x}^{2} P_{\left\lfloor\frac{m}{2}\right\rfloor-1}^{(\alpha+1, \beta)}(t) \Phi^{(0)}\right], \quad m \geqslant 2, \\
\tilde{\Psi}_{l m}^{(2)}= & \mu_{0} \bar{x}^{m-2} L_{l}^{-\beta}\left(a r^{2}\right)\left[P_{\left\lfloor\frac{m}{2}\right\rfloor-1}^{(\alpha+2, \beta)}(t)\left(\tilde{\Phi}^{(2)}-2 \bar{x} \Phi^{(1)}+\bar{x}^{2} \Phi^{(0)}\right)\right. \\
& \left.+\frac{2(\alpha+1)}{\left(2\left\lfloor\frac{m-1}{2}\right\rfloor+1\right)(N-1)} \bar{x}^{2} P_{\left\lfloor\frac{m}{2}\right\rfloor-1}^{(\alpha+1, \beta)}(t) \Phi^{(0)}\right], \quad m \geqslant 2, \\
\hat{\Psi}_{l m}^{(3)}= & \mu_{0} \bar{x}^{m-3} L_{l}^{-\beta}\left(a r^{2}\right)\left[P_{\left\lfloor\frac{m-3}{2}\right\rfloor}^{(\alpha+3, \beta)}(t)\left(\hat{\Phi}^{(3)}-2 \bar{x} \Phi^{(2)}+2 \bar{x}^{2} \Phi^{(1)}-\frac{2}{3} \bar{x}^{3} \Phi^{(0)}\right)\right. \\
& +\frac{2 \bar{x}^{2}}{2\left\lfloor\frac{m}{2}\right\rfloor-1} P_{\left\lfloor\frac{m-3}{2}\right\rfloor}^{(\alpha+2, \beta)}(t)\left(2(\alpha+2) \Phi^{(1)}-\bar{x} \Phi^{(0)}\right) \\
& \left.-\frac{2(2 \alpha+3)}{m(m-2)} \varepsilon(m) \bar{x}^{3} P_{\frac{m-3}{2}}^{(\alpha+1, \beta)}(t) \Phi^{(0)}\right], \quad m \geqslant 3 .
\end{aligned}
$$

In the previous formulas $\lfloor\cdot\rfloor$ denotes the integer part and $\varepsilon(m)=\frac{1}{2}\left(1-(-1)^{m}\right)$ is the parity of $m$. The spin functions $\Phi^{(k)}, \tilde{\Phi}^{(2)}, \hat{\Phi}^{(3)}$ (cf. Eqs. (36) and (37)) are built from a state $|s\rangle$ symmetric under $S_{12}$ and belonging to $\Sigma^{\prime}$ for $\tilde{\Psi}_{l m}^{(2)}$, and antisymmetric under $S_{12}$ for $\hat{\Psi}_{l m}^{(3)}$. The generalized Laguerre polynomials $L_{l}^{-\beta}$ and the Jacobi polynomials $P_{k}^{(\gamma, \beta)}$ appearing in Eqs. (A.1)-(A.5) are 
defined as

$$
\begin{aligned}
& L_{l}^{-\beta}(z)=\sum_{j=0}^{l}(-1)^{j}\left(\begin{array}{c}
l-\beta \\
l-j
\end{array}\right) \frac{z^{j}}{j !} \\
& P_{k}^{(\gamma, \beta)}(z)=\frac{1}{k !} \sum_{j=0}^{k} \frac{1}{2^{j} j !}(-k)_{j}(\gamma+\beta+k+1)_{j}(\gamma+j+1)_{k-j}(1-z)^{j},
\end{aligned}
$$

where

$$
(x)_{j}=x(x+1) \cdots(x+j-1)
$$

is the Pochhammer symbol. The eigenfunctions of type (A.5) are independent of the remaining ones (A.1)-(A.4) only for spin greater than 1/2 $(n>2)$, cf. [35]. The model (7) possesses two additional families of eigenfunctions (also derived in the previous reference), which have not been listed above as they do not yield any further eigenstates of the chain (5).

Let us now discuss the behavior of the terms $L_{l}^{-\beta}\left(a r^{2}\right)$ and $\bar{x}^{2 k} P_{k}^{(\alpha+i, \beta)}(t)$ in Eqs. (A.1)-(A.4) as $a \rightarrow \infty$. Consider first the polynomial $L_{l}^{-\beta}\left(a r^{2}\right)$. Since

$$
\left(\begin{array}{l}
l-\beta \\
l-j
\end{array}\right)=\frac{(N a)^{l-j}}{(l-j) !}\left(1+O\left(a^{-1}\right)\right),
$$

it follows that

$$
a^{-l} L_{l}^{-\beta}\left(a r^{2}\right)=\frac{\left(N-r^{2}\right)^{l}}{l !}+O\left(a^{-1}\right),
$$

where the term $O\left(a^{-1}\right)$ in the previous equation is actually a polynomial in $a^{-1}$. (Throughout this paper, the symbol $O\left(a^{-k}\right)$ denotes any function $f(a)$ such that $a^{k} f(a)$ has a finite (possibly zero) limit as $a \rightarrow \infty$.) For the other type of term, note that

$$
\begin{aligned}
\bar{x}^{2 k} P_{k}^{(\alpha+i, \beta)}(t)= & \frac{1}{k !} \sum_{j=0}^{k}\left[\frac{(-k)_{j}}{N^{j} j !}(\alpha+\beta+i+k+1)_{j}\right. \\
& \left.\times(\alpha+i+j+1)_{k-j} \bar{x}^{2(k-j)}\left(N \bar{x}^{2}-r^{2}\right)^{j}\right]
\end{aligned}
$$

is clearly a polynomial in $\mathbf{x}$. Taking into account that

$$
(\alpha+i+j+1)_{k-j}=(N a)^{k-j}\left(1+O\left(a^{-1}\right)\right),
$$

we obtain

$$
a^{-k} \bar{x}^{2 k} P_{k}^{(\alpha+i, \beta)}(t)=\frac{N^{k}}{k !} \bar{x}^{2 k}+O\left(a^{-1}\right),
$$

where $O\left(a^{-1}\right)$ is polynomial in $a^{-1}$ and $\mathbf{x}$.

\section{Appendix B. The method of moments}

The method of moments $[40,41]$ is a powerful tool for computing the density of eigenvalues of a large Hermitian matrix whose spectrum is not known explicitly. The method is based on the 
relation between a probability distribution $g(x)$, i.e., a nonnegative function whose integral over its support $\left[x_{1}, x_{2}\right]$ is one, and its resolvent

$$
R(z)=\int_{x_{1}}^{x_{2}} \frac{g(x)}{z-x} \mathrm{~d} x, \quad \operatorname{Im} z \neq 0 .
$$

Using the well-known identity

$$
\lim _{\epsilon \rightarrow 0+} \frac{\epsilon}{\epsilon^{2}+x^{2}}=\pi \delta(x),
$$

it is straightforward to show that

$$
g(x)=\mp \frac{1}{\pi} \lim _{\epsilon \rightarrow 0+} R(x \pm \mathrm{i} \epsilon), \quad x \in\left(x_{1}, x_{2}\right) .
$$

The previous formula makes it possible to compute the probability density $g(x)$ if the resolvent $R(z)$ is known. One of the key ingredients of the method is the fact that if $g$ is positive in a set of nonzero measure, the resolvent can be expanded as a continued fraction

$$
R(z)=\frac{1}{z-b_{0}-\frac{c_{1}}{z-b_{1}-\cdots}},
$$

where $b_{k}, c_{k}$ are the coefficients in the three-term recursion relation

$$
P_{k+1}(x)=\left(x-b_{k}\right) P_{k}(x)-c_{k} P_{k-1}(x) \quad\left(P_{-1}=0, P_{0}=1, c_{0}=1\right)
$$

satisfied by the orthogonal polynomial system $\left\{P_{k}(x)\right\}_{k=0}^{\infty}$ associated with the density $g(x)$. The polynomials $P_{k}$ are the monic polynomials determined by the orthogonality condition

$$
\left\langle P_{k} P_{l}\right\rangle \equiv \int_{x_{1}}^{x_{2}} P_{k}(x) P_{l}(x) g(x) \mathrm{d} x=0, \quad k \neq l .
$$

It can be shown [51] that these polynomials satisfy a three-term recursion relation of the form (B.3) with coefficients $b_{k}$ and $c_{k}$ given by

$$
b_{k}=\frac{\left\langle x P_{k}^{2}\right\rangle}{\left\langle P_{k}^{2}\right\rangle}, \quad c_{k}=\frac{\left\langle P_{k}^{2}\right\rangle}{\left\langle P_{k-1}^{2}\right\rangle} .
$$

In this paper we are interested in computing the density of eigenvalues (normalized to unity) of a Hermitian $d \times d$ matrix $\mathcal{A}$, i.e.,

$$
g(x)=\frac{1}{d} \sum_{i=1}^{d} \delta\left(x-\lambda_{i}\right),
$$

where $\lambda_{1} \leqslant \cdots \leqslant \lambda_{d}$ are the eigenvalues of $\mathcal{A}$. The corresponding resolvent

$$
R(z)=\frac{1}{d} \sum_{i=1}^{d}\left(z-\lambda_{i}\right)^{-1}=\frac{1}{d} \operatorname{tr}(z-\mathcal{A})^{-1}
$$

is thus a rational function of $z$. In this case the resolvent can also be expanded as a continued fraction of the form (B.2) terminating at level $d$, i.e., $c_{d}=0$ and Eqs. (B.3) and (B.4) hold for $k \leqslant d-1$. 
If the density $g$ is not known, the coefficients $b_{k}$ and $c_{k}$ cannot be computed from Eq. (B.4), and hence the expansion (B.2) cannot be used directly to evaluate the resolvent. On the other hand, the coefficients $b_{k}$ and $c_{k}$ can be determined once the moments $\left\langle x^{k}\right\rangle$ of the distribution $g$ are known. Another central idea of the method consists in replacing $\left\langle x^{k}\right\rangle$ by the average of the expectation values $\left(\mathbf{v}, \mathcal{A}^{k} \mathbf{v}\right)$ over a suitable set of normalized random vectors $\mathbf{v} \in \mathbb{R}^{d}$. More precisely, let $u_{i}, i=1, \ldots, d$, be $d$ independent random variables uniformly distributed in the interval $[-1,1]$, and let $\mathbf{v}$ be the vector with components $v_{i}=u_{i} /\|\mathbf{u}\|$. Clearly, the components of $\mathbf{v}$ satisfy

$$
\overline{v_{i} v_{j}}=\frac{1}{d} \delta_{i j}
$$

where the bar stand for the average over the random numbers $u_{i}$. We thus have

$$
\begin{aligned}
\overline{\left(\mathbf{v}, \mathcal{A}^{k} \mathbf{v}\right)} & =\sum_{i, j=1}^{d}\left(\mathcal{A}^{k}\right)_{i j} \overline{v_{i} v_{j}}=\frac{1}{d} \sum_{i=1}^{d}\left(\mathcal{A}^{k}\right)_{i i}=\frac{1}{d} \operatorname{tr}\left(\mathcal{A}^{k}\right)=\frac{1}{d} \sum_{i=1}^{d} \lambda_{i}^{k} \\
& =\frac{1}{d} \sum_{i=1}^{d} \int x^{k} \delta\left(x-\lambda_{i}\right) \mathrm{d} x=\left\langle x^{k}\right\rangle .
\end{aligned}
$$

The previous equality implies that

$$
\langle P(x)\rangle=\overline{(\mathbf{v}, P(\mathcal{A}) \mathbf{v})}
$$

where $P$ is an arbitrary polynomial. From Eq. (B.4) we immediately obtain the following formula for the coefficients $b_{k}$ and $c_{k}$ :

$$
b_{k}=\frac{\overline{\left(\mathbf{w}_{k}, \mathcal{A} \mathbf{w}_{k}\right)}}{\overline{\left\|\mathbf{w}_{k}\right\|^{2}}}, \quad c_{k}=\frac{\overline{\left\|\mathbf{w}_{k}\right\|^{2}}}{\overline{\left\|\mathbf{w}_{k-1}\right\|^{2}}},
$$

where $\mathbf{w}_{k} \equiv P_{k}(\mathcal{A}) \mathbf{v}$. By Eq. (B.3), the vectors $\mathbf{w}_{k}$ satisfy the recursion relation

$$
\mathbf{w}_{k+1}=\left(\mathcal{A}-b_{k}\right) \mathbf{w}_{k}-c_{k} \mathbf{w}_{k-1} \quad\left(\mathbf{w}_{-1}=0, \mathbf{w}_{0}=\mathbf{v}, c_{0}=1\right) .
$$

The latter formula, together with Eq. (B.7), can be used to recursively compute the coefficients $b_{k}$ and $c_{k}$ for all $k=1, \ldots, d-1$.

The number $d=n^{N}$ is typically very large, so that it is not feasible to compute all the coefficients $b_{k}$ and $c_{k}$ by the procedure just outlined. In practice, one only computes the first few of these coefficients, say up to $k=k_{0}$, and tries to estimate the part $T_{k_{0}}(z)$ of the continued fraction (B.2) involving the remaining coefficients. In many cases of interest [52], the coefficients $b_{k}$ and $c_{k}$ approximately stabilize for a wide range of values of $k$, namely $b_{k} \simeq b$ and $c_{k} \simeq c$ for $k_{0}<k<k_{1}<d$, where $k_{1} \gg k_{0}$. In this case the remainder $T_{k_{0}}(z)$ can be approximated as follows:

$$
T_{k_{0}}(z) \simeq \frac{c}{z-b-\frac{c}{z-b-\cdots}} \equiv T(z) .
$$

But the truncation factor $T(z)$ satisfies

$$
T(z)=\frac{c}{z-b-T(z)},
$$


so that

$$
T(z)=\frac{1}{2}\left(z-b \pm \sqrt{(z-b)^{2}-4 c}\right) .
$$

We thus obtain the following approximate formula for the resolvent:

$$
R(z) \simeq \frac{1}{z-b_{0}-\frac{c_{1}}{x-b_{1}-} \cdot-\frac{c_{k_{0}}}{z-b_{k_{0}}-T(z)}} .
$$

The previous equation can be written as

$$
R(z) \simeq \frac{A(z) \pm \mathrm{i} B(z) \sqrt{\omega(z)}}{C(z) \pm \mathrm{i} D(z) \sqrt{\omega(z)}},
$$

with $A, B, C, D$ polynomials in $z$ with real coefficients and

$$
\omega(z)=\left(z-x_{1}\right)\left(x_{2}-z\right), \quad x_{1,2}=b \pm 2 \sqrt{c} ;
$$

note that $x_{1,2} \in \mathbb{R}$, on account of Eq. (B.7). The RHS of (B.10) has a branch cut on the interval $\left[x_{1}, x_{2}\right]$, which is the support of its corresponding density function. From Eqs. (B.1) and (B.10) we finally obtain the following approximate formula for the normalized density of eigenvalues of the Hermitian matrix $\mathcal{A}$ :

$$
g(x) \simeq \frac{|A(x) D(x)-B(x) C(x)|}{C(x)^{2}+D(x)^{2} \omega(x)} \frac{\sqrt{\omega(x)}}{\pi}, \quad x \in\left[x_{1}, x_{2}\right] .
$$

Since the RHS of the previous equation cannot vanish identically in any subinterval of $\left[x_{1}, x_{2}\right]$ with nonzero length, it follows that the formula just obtained is only appropriate when the spectrum of $\mathcal{A}$ has no gaps.

\section{References}

[1] J.A. Minahan, K. Zarembo, JHEP 0303 (2003) 013.

[2] D. Berenstein, S.A. Cherkis, Nucl. Phys. B 702 (2004) 49.

[3] R. Roiban, A. Volovich, JHEP 0409 (2004) 032.

[4] N. Beisert, M. Staudacher, Nucl. Phys. B 727 (2005) 1.

[5] L. Freyhult, C. Kristjansen, T. Månsson, JHEP 0512 (2005) 008.

[6] A.S. Gorsky, Theor. Math. Phys. 142 (2005) 153.

[7] W. Heisenberg, Z. Phys. 49 (1928) 619.

[8] H. Bethe, Z. Phys. 71 (1931) 205.

[9] L. Hulthén, Ark. Mat. Astron. Fys. 26A (1938) 1.

[10] J. des Cloizeaux, J.J. Pearson, Phys. Rev. 128 (1962) 2131.

[11] H.M. Babujian, Phys. Lett. A 90 (1982) 479.

[12] L.A. Takhtajan, Phys. Lett. A 87 (1982) 479.

[13] C.K. Majumdar, D.K. Ghosh, J. Math. Phys. 10 (1969) 1388.

[14] I. Affleck, T. Kennedy, E.H. Lieb, H. Tasaki, Phys. Rev. Lett. 59 (1987) 799.

[15] F.D.M. Haldane, Phys. Rev. Lett. 60 (1988) 635.

[16] B.S. Shastry, Phys. Rev. Lett. 60 (1988) 639.

[17] J. Hubbard, Proc. R. Soc. London, Ser. A 276 (1963) 238.

[18] M.C. Gutzwiller, Phys. Rev. Lett. 10 (1963) 159.

[19] F. Gebhard, D. Vollhardt, Phys. Rev. Lett. 59 (1987) 1472.

[20] B. Sutherland, Phys. Rev. A 4 (1971) 2019.

[21] B. Sutherland, Phys. Rev. A 5 (1972) 1372. 
[22] Z.N.C. Ha, F.D.M. Haldane, Phys. Rev. B 46 (1992) 9359.

[23] K. Hikami, M. Wadati, J. Phys. Soc. Jpn. 62 (1993) 469.

[24] J.A. Minahan, A.P. Polychronakos, Phys. Lett. B 302 (1993) 265.

[25] A.P. Polychronakos, Phys. Rev. Lett. 70 (1993) 2329.

[26] F. Calogero, J. Math. Phys. 12 (1971) 419.

[27] F. Calogero, Lett. Nuovo Cimento 20 (1977) 251.

[28] H. Frahm, J. Phys. A: Math. Gen. 26 (1993) L473.

[29] A.P. Polychronakos, Nucl. Phys. B 419 (1994) 553.

[30] F. Finkel, A. González-López, Phys. Rev. B 72 (2005) 174411.

[31] D. Bernard, M. Gaudin, F.D.M. Haldane, V. Pasquier, J. Phys. A: Math. Gen. 26 (1993) 5219.

[32] A. Enciso, F. Finkel, A. González-López, M.A. Rodríguez, Nucl. Phys. B 707 (2005) 553.

[33] B. Basu-Mallick, N. Bondyopadhaya, Nucl. Phys. B 757 (2006) 280.

[34] A. Enciso, F. Finkel, A. González-López, M.A. Rodríguez, Phys. Lett. B 605 (2005) 214.

[35] A. Enciso, F. Finkel, A. González-López, M.A. Rodríguez, J. Phys. A: Math. Theor. 40 (2007) 1857.

[36] A.V. Turbiner, Commun. Math. Phys. 118 (1988) 467.

[37] M.A. Shifman, Int. J. Mod. Phys. A 4 (1989) 2897.

[38] A.G. Ushveridze, Quasi-Exactly Solvable Models in Quantum Mechanics, Institute of Physics Publishing, Bristol, 1994.

[39] G. Auberson, S.R. Jain, A. Khare, J. Phys. A: Math. Gen. 34 (2001) 695.

[40] C. Benoit, E. Royer, G. Poussigue, J. Phys.: Condens. Matter 4 (1992) 3125.

[41] J.L. Alonso, L.A. Fernández, F. Guinea, V. Laliena, V. Martín-Mayor, Phys. Rev. B 63 (2001) 054411.

[42] I.S. Gradshteyn, I.M. Ryzhik, Table of Integrals, Series, and Products, sixth ed., Academic Press, San Diego, 2000.

[43] J.M. Blair, C.A. Edwards, J.H. Johnson, Math. Comp. 30 (1976) 827.

[44] F. Calogero, A.M. Perelomov, Lett. Nuovo Cimento 23 (1978) 650.

[45] D. Dominici, math.CA/0601078.

[46] B. Simon, Ann. Inst. H. Poincaré, Sect. A (N.S.) 38 (1983) 295.

[47] D. Holcman, I. Kupka, Forum Math. 18 (2006) 445.

[48] A. Khare, I. Loris, R. Sasaki, J. Phys. A: Math. Gen. 37 (2004) 1665.

[49] F. Finkel, D. Gómez-Ullate, A. González-López, M.A. Rodríguez, R. Zhdanov, Commun. Math. Phys. 221 (2001) 477.

[50] F. Finkel, D. Gómez-Ullate, A. González-López, M.A. Rodríguez, R. Zhdanov, Nucl. Phys. B 613 (2001) 472.

[51] T.S. Chihara, An Introduction to Orthogonal Polynomials, Gordon and Breach, New York, 1978.

[52] P. Turchi, F. Ducastelle, G. Tréglia, J. Phys. C: Solid State Phys. 15 (1982) 2891. 